\title{
Theory for the reorientational dynamics in glass-forming liquids
}

\author{
T. Franosch, M. Fuchs, W. Götze, M. R. Mayr, and A. P. Singh \\ Physik Department, Technische Universität München, 85747 Garching, Germany
}

(Received 19 May 1997)

\begin{abstract}
The mode-coupling theory for ideal liquid-glass transitions is extended so that the structural relaxation for the reorientational degrees of freedom of a linear molecule, which is immersed in a system of spherical particles, can be described. Closed equations of motion for the correlation functions formed with tensor density fluctuations are derived, which deal with the molecule's translational and reorientational motion. From these equations the nonergodicity parameters of a hard dumbbell molecule are calculated, which quantify its arrest in a hard-sphere glass. For top-down symmetric molecules it is shown that the odd-angular-momentum variables can exhibit an ergodic-to-nonergodic transition, characterized by a continuous increase of the EdwardsAnderson parameters near the critical points. [S1063-651X(97)03011-0]

PACS number(s): 64.70.Pf, 61.20.Lc
\end{abstract}

\section{INTRODUCTION}

Glass-forming liquids exhibit structural-relaxation dynamics if they are cooled or compressed. There are two outstanding features of this glass-transition precursor [1]. The first is the strong sensitivity on temperature $T$ or density $n$ of the characteristic time scale $\tau$ for the slowest process, called the $\alpha$ relaxation. The second is the stretching of decay curves or susceptibility spectra over large windows of time $t$ or frequency $\omega$, respectively. The classical experimental studies, which have been carried out for more than a century now, deal with structural relaxation on time scales of order $10^{-8} \mathrm{~s}$ or larger. This work did not lead to a coherent physical picture, let alone a theory, of these fascinating dynamical phenomena in condensed disordered matter.

The time scale for normal liquid dynamics is about $10^{-13} \mathrm{~s}$. On this scale neither liquids nor glasses exhibit structural relaxation as is known from, for example, Raman spectroscopy or neutron scattering and molecular-dynamicssimulation work [2-4]. Consequently, structural relaxation has to evolve within the gigahertz or picosecond bands, and one can expect that studies of these dynamical windows reveal the secrets of the indicated phenomena. Great efforts and progress have been made towards this goal during the past ten years. We only mention some very recent achievements: measurements of the depolarized-light-scattering spectra of orthoterphenyl (OTP) over a four-decade frequency window [5], light-scattering [6] and neutronscattering [7] spectroscopy for glycerol, impulsivestimulated-light-scattering work for supercooled Salol [8,9], and $0.4 \mathrm{Ca}\left(\mathrm{NO}_{3}\right)_{2} 0.6 \mathrm{KNO}_{3}(\mathrm{CKN})[10]$, transient hole burning for propylene carbonate [11], dielectric-loss spectroscopy within the full gigahertz band for glycerol [12] and CKN [13], and molecular-dynamics simulations of $\mathrm{Ni}_{80} \mathrm{P}_{20}[14,15]$, water [16], and OTP [17]. Citations of the earlier work can be found in Refs. [5-17]. A series of properties of structural relaxation has been discovered, which has not been observed in the classical glass-transition research. Some of them can be directly identified by a look at the raw data: the appearance of two fractal power laws for the spectra $[6,7,18,19]$ or for the equivalent decay curves [14-16], unconventional scaling laws for the dynamics in a window between the cen- ter of the $\alpha$ process and the short-time normal liquid dynamics $[11-13,20]$, and a square-root anomaly for the $\alpha$-peak intensity $[8-10,21]$. The mentioned modern studies support an old conjecture by Goldstein [22] concerning the significance of a characteristic temperature $T_{c}$ located in the supercooled region above the calorimetric glass-transition temperature $T_{g}$. For $T>T_{c}$, or for $n$ smaller than an analogous characteristic density $n_{c}$, transport phenomena are connected with the rattling of particles in self-consistently maintained cages and with the cooperative motion as manifested by the building of backflow patterns. For $T<T_{c}$ or $n>n_{c}$, on the other hand, the particles are almost arrested in a spontaneously frozen effective-free-energy landscape and transport is triggered by thermally activated hopping of the particles over saddle points.

Of particular relevance for establishing the mentioned picture are the structural-relaxation studies for dense colloidal suspensions [23-26]. Within the accessible dynamical window of up to eight orders of magnitude, a sharp transition from an ergodic liquid for $n<n_{c}$ to a nonergodic glass for $n>n_{c}$ is observed. The static structure varies smoothly with changes of density for $n$ near $n_{c}$, and it reflects nothing but intermediate-range order due to random packing. For $n>n_{c}$ no hopping effects have been observed. The detailed measurements of the decay curves for density fluctuations exhibit the same time fractals, scaling laws, and square-root anomalies as mentioned above for conventional systems.

The above cited experiments [5-26] are compatible with the conclusion that structural relaxation is a dynamical phenomenon connected with a glass-transition singularity. The singularity is specified by critical values of control parameters like $T_{c}$ and $n_{c}$, which separate ergodic liquid from nonergodic glass states. The reality differs from the specified idealization; due to hopping processes the singularity is avoided. The escape of the system from one of the many free-energy valleys to a new one appears as an instability of the nonergodic state. This causes the $\alpha$ process for $T_{g}<T$ $<T_{c}$, which deals with the decay of the otherwise expected spontaneously arrested glass structure. The properties of the idealized $T_{c}$ transition scenario remain valid for times short compared to $\tau$.

Let us consider a system of $N$ identical particles whose 
center-of-mass positions are denoted by $\vec{r}^{\kappa}, \kappa=1, \ldots, N$. The simplest variables dealing with structure are the density fluctuations as a function of wave vector $\vec{q}: \varrho(\vec{q})$ $=\Sigma_{\kappa} \exp \left(\mathrm{i} \vec{q} \cdot \vec{r}^{\kappa}\right)$. The structure factor $S(q)=\left\langle|\varrho(\vec{q})|^{2}\right\rangle / N$ provides the simplest information on the particle distribution. Here and in the following \langle\rangle denotes canonical averaging and $q=|\vec{q}|$ indicates a vector modulus. The simplest functions dealing statistically with structure dynamics are the density correlators $\Phi_{q}(t)=\left\langle\varrho(\vec{q}, t)^{*} \varrho(\vec{q})\right\rangle /\left\langle|\varrho(\vec{q})|^{2}\right\rangle$. They are measured, for example, in the cited colloid experiments [23-25]. Within the Zwanzig-Mori formalism one can derive the equation of motion [2-4]

$$
\partial_{t}^{2} \Phi_{q}(t)+\Omega_{q}^{2} \Phi_{q}(t)+\int_{0}^{t} M_{q}(t-\bar{t}) \partial_{\bar{t}} \Phi_{q}(\bar{t}) d \bar{t}=0 .
$$

Here $\Omega_{q}=q v / \sqrt{S(q)}$, with $v$ denoting the thermal velocity, is the well-known characteristic frequency, quantifying the initial decay of density correlations: $\Phi_{q}(t)=1-\Omega_{q}^{2} t^{2} / 2$ $+O\left(t^{3}\right)$. The kernel $M_{q}(t)$ describes correlations of fluctuating forces. If one is interested in a theory of structural relaxation, it is suggestive to split the kernel in a regular part $M_{q}^{\mathrm{reg}}(t)$, dealing with the transient dynamics, and a remain$\operatorname{der} \Omega_{q}^{2} m_{q}(t)$,

$$
M_{q}(t)=M_{q}^{\mathrm{reg}}(t)+\Omega_{q}^{2} m_{q}(t) .
$$

The latter is supposed to deal with the slowly fluctuating parts of the forces, which are caused by the slowly relaxing structure. Extending the original theories of the cage effect in simple liquids [2-4] one can motivate for $m_{q}(t)$ a representation as mode-coupling functional,

$$
m_{q}(t)=\mathcal{F}_{q}\left(\Phi_{k}(t)\right)
$$

Here $\mathcal{F}_{q}$ is a quadratic polynomial

$$
\mathcal{F}_{q}\left(\widetilde{f}_{k}\right)=\sum_{\vec{k}+\vec{p}=\vec{q}} V(\vec{q} ; \vec{k}, \vec{p}) \widetilde{f}_{k} \widetilde{f}_{p}
$$

where the vertices $V(\vec{q} ; \vec{k}, \vec{p})$ are specified in terms of the structure factor [27]. The latter can be evaluated for simple liquids with some standard approximations [3]. Thereby the vertices enter the theory as coupling constants; they are known smooth functions of $T$ and $n$. For $M_{q}^{\text {reg }}(t)$ some established model can be used; in a most simple approximation it may be ignored altogether; a more reasonable approximation is the replacement of $\int_{0}^{t} M_{q}^{\mathrm{reg}}\left(t-t^{\prime}\right) \partial_{t^{\prime}} \Phi_{q}\left(t^{\prime}\right) d t^{\prime}$ by a friction term $\nu_{q} \partial_{t} \Phi_{q}(t)$. As a result one gets a closed set of regular nonlinear equations of motion for the evaluation of the correlators. These Eqs. (1) are the basis of the modecoupling theory (MCT) of structural relaxation [28]. The original derivation of the MCT is reviewed in Ref. [29] and a critical reconsideration of the underlying approximations was given recently [30] within a perturbation-theoretical formalism.

The MCT equations of motion exhibit a bifurcation for the long-time limits

$$
\Phi_{q}(t \rightarrow \infty)=f_{q} .
$$

If a control parameter, say $n$, is smaller than a critical value $n_{c}$, the correlators relax towards the equilibrium value $f_{q}$ $=0$, as expected for an ergodic liquid. However, for $n \geqslant n_{c}$ density fluctuations arrest in a disordered solid, quantified by a Debye-Waller factor $f_{q}>0$, also called nonergodicity parameter or glass form factor. Edwards and Anderson have pointed out in connection with a discussion of spin glasses [31] that the appearance of nonzero long-time limits like $f_{q}$ is the signature of ideal glass states. Therefore these limits are also called Edwards-Anderson parameters. At the critical point $n_{c}$ the long-time limits jump from zero to a nonzero critical value $f_{q}^{c}>0$. The $f_{q}$ are to be evaluated from the mode-coupling functional $\mathcal{F}$ via the equations $f_{q} /\left(1-f_{q}\right)$ $=\mathcal{F}_{q}\left(f_{k}\right)$ [28]. Thus, $n_{c}$ and $f_{q}$ are determined by the equilibrium structure factors $S(q)$; they are independent of the transient dynamics as specified by $\Omega_{q}$ and $M_{q}^{\text {reg }}(t)$. The bifurcation implies a dynamical scenario that is strikingly similar to what is discussed in many recent experiments, for example, in Refs. [5-26]. The qualitative features of the transition scenario can be understood by solving Eqs. (1) analytically by asymptotic expansions near the transition point. This mathematical work is explained comprehensively and illustrated in detail for a hard-sphere-liquid model in Ref. [32], where also the earlier theoretical papers are cited. The asymptotic solutions establish universality features of the MCT scenario for the evolution of structural relaxation [29]. These general results are important outcomes for an assessment of the MCT. The first comparisons of MCT results and experiments are reviewed in Refs. [33,34]. The theory based on Eqs. (1) overemphasizes the cage effect. In liquids there are phonons, which kick particles out of their self-trapping. If the couplings of fluctuating forces to phonons are incorporated, one gets the extended MCT, where the sharp transition at $T_{c}$ or $n_{c}$ is replaced by a smooth crossover [35-37]. One finds an $\alpha$ process also for $T<T_{c}$ or $n>n_{c}$, where $f_{q}$ specifies the intensity of this low-frequency part of the relaxation spectrum.

All results for the evolution of structural relaxation of the hard-sphere system, as they had been obtained by leadingorder asymptotic solutions of the corresponding MCT equations, have been compared by van Megen and Underwood with their measurements of $\Phi_{q}(t)$. The theory accounts for their data on a $15 \%$ accuracy level $[23,24,38,39]$. Consequently, the hard-sphere colloidal suspensions provide an example for which structural relaxation and the glass transition are understood quantitatively within a microscopic theory. The asymptotic solutions [32] provide an understanding of all the qualitative features of the evolution of structural relaxation identified so far for this system. MCT has been generalized to the treatment of mixtures of spherical molecules, and the calculated results for the functions that characterize the glass structure account well for the corresponding quantities obtained from molecular-dynamics simulations [40].

Tests of the MCT by data obtained for liquids of nonspherical molecules depend on the assumption that the experiments can be described by the universal features [29]. These are quantified here, as in other singularity theories, by a well-defined set of parameters like $n_{c}$ or $T_{c}$ and by amplitudes like $f_{q}^{c}$. These quantities enter the data analysis as fit parameters. The first complete test of the leading-order 
asymptotic results for the evolution of structural relaxation was done for depolarized-light-scattering spectra of CKN by Li et al. [20]. Also the extended MCT predicts universal scaling formulas [36,37], which complete the interpretation of the structural relaxation in, for example, CKN, Salol [41], or OTP [5]. There are a considerable number of further quantitative tests of the MCT that are reported and to a large extent cited in Refs. [5-26].

The indicated experimental findings provide an obvious motivation for an extension of the MCT to systems of nonspherical molecules. A more subtle motivation is given by the fact that the range of validity of the universal asymptotic results, like scaling laws or power laws for the spectra, depends on the probing variable. Consider, for example, the von Schweidler fractal decay for the hard-sphere system. It holds for a much larger dynamical window for those density fluctuations whose wave vector $q$ is located near the structure-factor-peak position than for $q$ near the structurefactor minimum [32]. It is plausible that the MCT scaling laws hold for a different temperature interval or frequency range for depolarized-light-scattering spectra than for, say, dielectric-loss data. To decide upon these issues one has to study the amplitudes that quantify the leading corrections to the leading asymptotic laws [32]. An understanding of reorientational relaxation, which goes beyond the justification of universality features, therefore requires an explicit formulation of the MCT for these processes, in particular, a quantification of the mode-coupling functionals.

In this paper, the simplest facet of the problems connected with glassy reorientational dynamics shall be analyzed. We will develop the MCT for the motion of a single rigid nonspherical molecule, which exhibits an axis of rotational symmetry $\vec{e}$. This molecule shall be immersed in a system of spherical particles. The most important functions describing reorientational motion are [3]

$$
C^{(j)}(t)=\left\langle P_{j}(\vec{e}(t) \cdot \vec{e})\right\rangle,
$$

where $P_{j}$ denotes the Legendre polynomial of degree $j$ $=1,2 \ldots$. Beginning with Debye's theory of dipole relaxation in liquids, these functions have been discussed extensively, and we refer the reader to the monograph by Evans et al. [42] for a review. If one considers a dilute solution of polar molecules in a dielectrically inert environment, the system's dielectric-loss spectrum is proportional to $\omega C^{(1) \prime \prime}(\omega)$. Here $C^{(j) \prime \prime}(\omega)$ denotes the Fourier cosine transform for frequency $\omega$ of $C^{(j)}(t)$. Similarly, the cross section for depolarized light scattering for the solution is proportional to $C^{(2) \prime \prime}(\omega)$. Thus the functions $C^{(j)}$ are of direct interest for the interpretation of experiments. The observed puzzling time dependence of $C^{(j)}(t)$ is due to the coupling of the five degrees of freedom of the test molecule to the ones of the molecules of the surrounding system. The surroundings cause forces, which lead to librations and to relaxation phenomena. The molecule's motion reflects the surroundings' dynamics. The approximation schemes for $C^{(j)}(t)$, which have been discussed in the past, did not lead to an acceptable description of the measured structural relaxation phenomena [42], since no adequate theories for the dynamics of the glassy environment had been available. MCT offers a way out of this problem, since the solutions of Eqs. (1) can be used as input information. Polarized light scattering and neutron scattering experiments probe a combination of translational and reorientational motion of the molecules. MCT provides a formalism defining how the structure relaxation of the various degrees of freedom are related, in particular, how they enter the measured spectra.

In a normal liquid the $C^{(j)}(t)$ relax to zero for long times [42]. But within an ideal glass environment, one expects spontaneous arrest for reorientational motion, i.e., positive long-time limits $f_{j}$ in analogy to Eq. (2);

$$
C^{(j)}(t \rightarrow \infty)=f_{j} .
$$

The $f_{j}$ correspond to the Edwards-Anderson parameters, introduced in the theory of spin glasses [31]. Within spin-glass theories one usually introduces quenched disorder in an $a d$ hoc manner and then studies the most favorable states compatible with the spin-spin interactions. In our approach the frozen disorder is calculated with Eqs. (1) and then the motion of the test molecule in the cages, formed by its neighbors, will be studied.

The scope of the intended broadening of the modecoupling approach towards handling nonsimple liquids is too narrow to lead to a complete theory of systems like the cited Salol or glycerol. The molecules in those systems do not have an axis of rotational symmetry $\vec{e}$. It will be obvious, however, that our work can be extended to a treatment of general rigid molecules with three rotational degrees of freedom. The functions that will be constructed in the following with spherical harmonics have to be extended to the Wigner functions $[43,44]$. The serious problem for a complete MCT of molecular liquids is the need to derive matrix generalizations of the Eqs. (1) so that coherent fluctuations of reorientations can be handled. Such work has been done recently by Schilling and Scheidsteger [45]. The form factors shown in their work describe the interplay of hard-sphere repulsions with long-range dipole interactions. The numerical results that we are going to discuss for the glass form factors deal with the dynamics of reorientations as it is dominated by steric hindrance.

The present work is arranged as follows. In Sec. II the irreducible tensor densities and the corresponding currents are introduced, which will be needed for the description of the molecule's dynamics. The exact equations of motion will be formulated for the density correlation functions, where the interaction effects enter via fluctuating-force correlation kernels. Then (Sec. III) the equations will be closed by a modecoupling approximation. As a result, a nonlinear-dynamics model for the molecule's motion will be obtained, describing structural relaxation. In particular, equations for the Edwards-Anderson parameters will be derived. In Sec. IV quantitative results for the glass form factors will be presented and discussed for a hard dumbbell molecule immersed in a hard-sphere glass.

\section{BASIC CONCEPTS AND EQUATIONS}

\section{A. Density and momentum density fluctuations}

In this section we define the variables that will be used for the description of the molecule's dynamics. Using the conventional terminology [46], variable $A, B$, etc. are consid- 
ered as vectors in the state space with a scalar product $(A \mid B)=\left\langle A^{*} B\right\rangle$. The time evolution is generated by the Hermitian Liouvillian $\mathcal{L}: A(t)=\exp (\mathrm{i} \mathcal{L} t)$. Hence $\partial_{t} A=\mathrm{i} \mathcal{L} A$ $=\{H, A\}$, where $H$ is the Hamiltonian and $\{$,$\} denotes the$ Poisson brackets. The Hamiltonian is the sum of the Hamiltonian for the surroundings $H_{S}$, that for the moleculesurroundings interaction potential $H_{\mathrm{int}}$, and that for the rigid molecule's kinetic energy $H_{0}$. The latter is the sum of translational and rotational parts: $H_{0}=H_{\mathrm{T}}+H_{\mathrm{R}}$. If $\vec{r}$ denotes the center of mass and $\vec{p}$ the momentum, $H_{\mathrm{T}}=p^{2} /\left(2 I_{\mathrm{T}}\right)$ and $\vec{p}$ $=I_{\mathrm{T}} \dot{\vec{r}}$, where the inertia parameter $I_{\mathrm{T}}$ for translations is the molecule's mass. If $F(\vec{r})$ is a function of position only, one finds $\mathrm{i} \mathcal{L} F(\vec{r}) I_{\mathrm{T}}=\vec{p} \cdot \partial F / \partial \vec{r}$. The molecule's orientation shall be specified by the solid angle $\Omega=(\phi, \theta),-\pi \leqslant \phi<\pi ; 0$ $\leqslant \theta \leqslant \pi$ for the axis of rotational symmetry $\vec{e}$. Instead of $\theta$ one can also use the projection of $\vec{e}$ on the $z$ axis: $\eta$ $=\cos \theta$. If $I_{\mathrm{R}}$ denotes the molecule's moment of inertia, one gets $H_{\mathrm{R}}=\left\{\left[p_{\phi}^{2} /\left(1-\eta^{2}\right)\right]+\left[p_{\eta}^{2}\left(1-\eta^{2}\right)\right]\right\} /\left(2 I_{\mathrm{R}}\right)$, where $p_{\phi}$, $p_{\eta}$ are the canonical momenta for $\phi$ and $\eta$, respectively. If $G(\vec{e})$ denotes a function of the orientation only, one calculates $\mathrm{i} \mathcal{L} G(\vec{e}) I_{\mathrm{R}}=\left[p_{\phi} /\left(1-\eta^{2}\right)\right] \partial G / \partial \phi+\left[p_{\eta}\left(1-\eta^{2}\right)\right] \partial G /$ $\partial \eta$. The momenta have a Gaussian distribution quantified by the thermal velocities $v_{\alpha}=\sqrt{\mathrm{T} / I_{\alpha}}$ for translation, $\alpha=\mathrm{T}$, and rotation, $\quad \alpha=\mathrm{R}, \quad$ respectively: $\quad\left\langle\left(p_{i} / I_{\mathrm{T}}\right)^{2}\right\rangle_{0}=v_{\mathrm{T}}^{2}$, $\left\langle\left(p_{\phi} / I_{\mathrm{R}}\right)^{2}\right\rangle_{0} /\left(1-\eta^{2}\right)=\left\langle\left(p_{\eta} / I_{\mathrm{R}}\right)^{2}\right\rangle_{0}\left(1-\eta^{2}\right)=v_{\mathrm{R}}^{2}$. Here \langle\rangle$_{0}$ denotes averaging for fixed orientations, and temperatures are measured in units of $k_{B}$.

A complete set of variables, specifying the molecule's position in its five-dimensional configuration space, are tensor density fluctuations for wave vector $\vec{q}$ and degree $j$ :

$$
\varrho_{j}^{\nu}(\vec{q})=R_{j}^{\nu} \exp (\overrightarrow{\mathrm{i} q} \cdot \vec{r}) .
$$

The orientational part $R_{j}^{\nu}$ is chosen proportional to the spherical harmonics $Y_{j}^{\nu}: R_{j}^{\nu}=i^{j} \sqrt{4 \pi} Y_{j}^{\nu}(\vec{e}), j=0,1, \ldots, \nu$ $=-j,-j+1, \ldots j$. The conventions follow Ref. [47] so that $\varrho_{j}^{\nu}(\vec{q})^{*}=(-1)^{\nu+j} \varrho_{j}^{-\nu}(-\vec{q})$. The variables $\varrho_{j}^{\nu}(\vec{q})$ have even parity under time inversion. The time derivatives of the densities split naturally into translational and reorientational parts:

$$
\mathcal{L} \varrho_{j}^{\nu}(\vec{q})=\sigma_{\mathrm{T} j}^{\nu}(\vec{q}) / I_{\mathrm{T}}+\sigma_{\mathrm{R} j}^{\nu}(\vec{q}) / I_{\mathrm{R}}
$$

The momentum density fluctuations, $\sigma_{\alpha j}^{\nu}(\vec{q})$, can be written similar to Eq. (5):

$$
\sigma_{\alpha j}^{\nu}(\vec{q})=S_{\alpha j}^{\nu} \exp (i \vec{q} \cdot \vec{r}), \quad \alpha=\mathrm{T}, \mathrm{R} .
$$

Here $S_{\mathrm{T} j}^{\nu}=\vec{q} \cdot \vec{p} R_{j}^{\nu}$ and $S_{\mathrm{R} j}^{\nu}=I_{\mathrm{R}} \mathcal{L} R_{j}^{\nu}$ can be easily evaluated as a function of $\vec{e}$ with the formulas noted in the preceding paragraph. The new variables obey $\sigma_{\alpha j}^{\nu}(\vec{q})^{*}$ $=-(-1)^{\nu+j} \sigma_{\alpha j}^{-\nu}(-\vec{q})$, and they are odd under time inversion.

Because of translational invariance, the specified fluctuations are pairwise orthogonal, unless the wave vectors are equal. A similar statement holds with respect to the tensor indices because of rotational symmetry. The density fluctuations are normalized:

$$
\left(\varrho_{i}^{\mu}(\vec{p}) \mid \varrho_{j}^{\nu}(\vec{k})\right)=\delta_{i j} \delta^{\mu \nu} \delta_{\vec{p} \vec{k}}
$$

The momentum densities are orthogonal to the densities since they have opposite time-inversion symmetry. The remaining averages can be reduced to $\left\langle(\vec{q} \vec{p})^{2}\right\rangle=\left(q v_{\mathrm{T}} I_{\mathrm{T}}\right)^{2}$ and to $\left\langle\left(1-\eta^{2}\right) p_{\eta}^{2}\left(\partial R_{i}^{0} / \partial \eta\right)\left(\partial R_{j}^{0} / \partial \eta\right)\right\rangle=I_{\mathrm{R}}^{2} v_{\mathrm{R}}^{2} j(j+1) \delta_{i j}$. As a result one finds

$$
\left(\sigma_{\alpha i}^{\mu}(\vec{p}) \mid \sigma_{\beta j}^{\nu}(\vec{k})\right)=\delta_{\alpha \beta} \delta_{i j} \delta^{\mu \nu} \delta_{\vec{p} \vec{k}} I_{\alpha}^{2} \Omega_{\alpha j}^{2}(p) .
$$

Here the $\Omega_{\alpha j}(q)$ are given by the characteristic frequencies for the free particle dynamics $[3,42]$.

$$
\Omega_{\mathrm{T} j}(q)=\Omega_{q}^{s}=v_{\mathrm{T}} q, \quad \Omega_{\mathrm{R} j}(q)=\Omega_{j}=v_{\mathrm{R}} \sqrt{j(j+1)} .
$$

\section{B. The molecule-surroundings-interaction functions}

The interactions between the molecule and the surrounding spherical particles will enter our theory via direct correlation functions $c_{J}(q)$. These real functions are defined by

$$
n S(q) c_{J}(q)=\left\langle\varrho\left(\vec{q}_{0}\right) * \varrho_{J}^{0}\left(\vec{q}_{0}\right)\right\rangle .
$$

Here and in the following $\vec{q}_{0}=(0,0, q)$ denotes a vector of length $q$ directed parallel to the $z$ axis. $\varrho(\vec{q})$ denotes the surroundings' density fluctuation and $S(q)$ is the surroundings' structure factor, introduced in Sec. I. The right-hand side (rhs) of Eq. (11) is the static solute-solvent structure factor $S_{J}(q)=n S(q) c_{J}(q)$. In the limit of weak interaction potentials one finds $n c_{J}(q)=-n v_{J}^{0}\left(\vec{q}_{0}\right) / T$, where $v_{J}^{0}\left(\vec{q}_{0}\right)$ is the coefficient of the decomposition in spherical harmonics of the molecule-surroundings-interaction-potential Fourier transform. Hence, $-n c_{J}(q)$ plays the role of an effective interaction potential. The fluctuations $\varrho(\vec{q})$ are irreducible tensor density fluctuations of degree $J=0$. Thus one derives from translational and rotational invariance the formula for the overlap of $\varrho(\vec{q})$ with the density fluctuations of the molecule

$$
\left\langle\varrho(\vec{p})^{*} \varrho_{J}^{M}(\vec{q})\right\rangle=\delta_{\vec{p} \vec{q}}[4 \pi /(2 J+1)]^{1 / 2} Y_{J}^{M}(\vec{p}) n S(p) c_{J}(p)
$$

The effective potentials also determine the overlaps of $\varrho(\vec{q})$ with three kinds of molecule pair modes, which will be needed in the following. These are denoted by $\pi(\alpha)$, $\alpha=\mathrm{O}, \mathrm{T}, \mathrm{R}$

$$
\pi_{j l}^{\mu \kappa}(\vec{q}, \vec{k}, \alpha)=\left\{\begin{array}{cc}
\varrho_{j}^{\mu}(\vec{q}) \varrho_{l}^{\kappa}(\vec{k}) & \text { if } \alpha=\mathrm{O} \\
-\mathrm{i}\left\{\sigma_{\alpha j}^{\mu}(\vec{q}), \varrho_{l}^{\kappa}(\vec{k})\right\} & \text { if } \alpha=\mathrm{T}, \mathrm{R} .
\end{array}\right.
$$

These pair modes can be written as a linear combination of fluctuations $\varrho_{J}^{M}(\vec{q}+\vec{k})$ if one expresses tensor products as 
linear combinations of irreducible tensors, with 3-j symbols and reduced averages determining the coefficients [47]. With Eq. (12) one obtains

$$
\begin{aligned}
\left\langle\varrho(\vec{p})^{*} \pi_{j l}^{\mu \kappa}(\vec{q}, \vec{k}, \alpha)\right\rangle= & \delta_{\vec{p}, \vec{q}+\vec{k}} \mathrm{i}^{j+l}[4 \pi(2 j+1)(2 l+1)]^{1 / 2} \\
& \times \sum_{J M} Y_{J}^{M}(\vec{p})^{*} n S(p) c_{J}(p) \\
& \times\left(\begin{array}{ccc}
j & l & J \\
\mu & \kappa & M
\end{array}\right) \\
& \times\left(\begin{array}{ccc}
j & l & J \\
0 & 0 & 0
\end{array}\right) \mathrm{i}^{-J} C_{\alpha}(j l J ; \vec{q} \cdot \vec{k})(14 \mathrm{a})
\end{aligned}
$$

$C_{\mathrm{O}}=1, \quad C_{\mathrm{T}}=\vec{q} \cdot \vec{k}, \quad C_{\mathrm{R}}=[J(J+1)-j(j+1)-l(l+1)] / 2$.

Because of reflection symmetry, tensor degrees are coupled so that $j+l+J$ is even. If a top-down symmetric molecule is considered there is a further symmetry of the system with respect to inversions of the axis $\vec{e}$. The functions $c_{J}$ vanish for odd $J$ in this case, and the overlaps in Eq. (14a) are nonzero only if $j+l$ is even.

\section{The density correlators for the molecule}

A statistical description of the molecule's motion in configuration space is given by the correlators $\left\langle\varrho_{i}^{\mu}(\vec{p}, t)^{*} \varrho_{j}^{\nu}(\vec{q})\right\rangle$. Because of translational invariance the overlaps for $\vec{p} \neq \vec{q}$ vanish so that one can write

$$
\left(\varrho_{i}^{\mu}(\vec{p}, t) \mid \varrho_{j}^{\nu}(\vec{q})\right)=\delta_{\vec{p} \vec{q}} \Phi_{i j}^{\mu \nu}(\vec{q}, t) .
$$

Let $\mathcal{D}(\vec{q})$ be a rotation of vector $\vec{q}_{0}=(00 q)$ into vector $\vec{q}$ and let $D_{\nu \kappa}^{j}(\vec{q})$ denote the Wigner functions for the corresponding irreducible representation of $\mathcal{D}(\vec{q})$ of degree $j$. If $(\phi, \theta, \psi)$ denote the three Euler angles, we write $D_{\nu \kappa}^{j}(\phi, \theta, \psi)$ $=\exp (\mathrm{i} \nu \phi+\mathrm{i} \kappa \psi) d_{\nu \kappa}^{j}(\theta)$, where the real quantities $d_{\nu \kappa}^{j}(\theta)$ are the reduced Wigner functions [47]. Rotational invariance implies a representation of general density correlators as combination of those for $\vec{q}_{0}$ :

$$
\begin{gathered}
\Phi_{i j}^{\mu \nu}(\vec{q}, t)=\sum_{\kappa} D_{\mu \kappa}^{i}(\vec{q})^{*} D_{\nu \kappa}^{j}(\vec{q}) \Phi_{i j}(q \kappa, t), \\
\Phi_{i j}(q \kappa, t)=\left(\varrho_{i}(q \kappa, t) \mid \varrho_{j}(q \kappa)\right), \quad \varrho_{i}(q \kappa)=\varrho_{i}^{\kappa}\left(\vec{q}_{0}\right) .
\end{gathered}
$$

The goal of every theory for the dynamics of a linear test molecule moving in a homogeneous isotropic environment is the evaluation of the infinite matrix $\Phi_{i j}(q \kappa, t) ; i, j$ $=0,1, \ldots ; \kappa=0, \pm 1, \ldots, \pm \min (i, j)$. The matrix elements are the correlation functions for tensor density fluctuations of wave-vector modulus $q$, degrees $i$ and $j$, and helicity $\kappa$. Correlations between variables of different helicities do not enter, since rotational invariance implies $\left(\varrho_{i}(q \mu t) \mid \varrho_{j}(q \nu)\right)$ $=0$ for $\mu \neq \nu$.
Equation (16a) holds for all directions of $\vec{q}$, if the limit $q \rightarrow 0$ is considered for the correlators. Therefore $\Phi_{i j}^{\mu \nu}(\overrightarrow{0}, t)$ $=\delta_{i j} \delta^{\mu \nu} \Sigma_{\kappa} \Phi_{j j}^{\kappa \kappa}(\overrightarrow{0}, t) /(2 j+1)$. Using the addition theorem for spherical harmonics the rhs is given by the functions defined in Eq. (3). The $C^{(j)}(t)$ are obtained as longwavelength limits

$$
\Phi_{i j}(q \rightarrow 0 \mu, t)=\delta_{i j} C^{(j)}(t) \quad \text { if }|\mu| \leqslant j .
$$

The cross section for the scattering of a test particle can be written as Fourier cosine transform of an intermediate scattering function $F(t)=\left\langle X(t)^{*} X\right\rangle$, with $X$ denoting the coupling variable [46]. For example, the incoherent neutron scattering in a simple liquid is given by the self-correlation function $\Phi_{q}^{s}(t)=\langle\exp [-\mathrm{i} \vec{q} \cdot \vec{r}(t)] \exp [\mathrm{i} \vec{q} \cdot \vec{r}]\rangle[2-4]$. The coupling variable for a neutron scattering process from a linear molecule for a momentum transfer $\vec{q}$ can be expressed in terms of form factors $b_{j}(q)$ as $X$ $=\exp (\overrightarrow{\mathrm{i}} \vec{q} \cdot \vec{r}) \Sigma_{j \nu} b_{j}(q) \sqrt{4 \pi /(2 j+1)} Y_{j}^{\nu}(\vec{q})^{*} R_{j}^{\nu}(\vec{e})$. If the molecule consists of $s$ atoms with scattering lengths $f_{\lambda}$ that are placed at $\vec{r}+\delta_{\lambda} \vec{e}, \quad \lambda=1, \ldots, s, \quad$ one gets $b_{l}(q)$ $=\Sigma_{\lambda} f_{\lambda} \sqrt{2 l+1} j_{l}\left(q \delta_{\lambda}\right)$, where $j_{l}$ denotes the spherical Bessel function. From Eq. (16a) one obtains

$$
F(t)=\sum_{i j} b_{i}(q) * \Phi_{i j}(q 0, t) b_{j}(q) .
$$

In order to calculate the scattering properties translational as well as rotational degrees of freedom have to be taken into account. Only for very small $q$ can one restrict the sum to the $i=j=0$ term and reduce the problem to a discussion of $\Phi_{q}^{s}(t)$.

The coefficients of a Taylor expansion with respect to $t$ in Eq. (16b) are given by matrix elements of the Liouvillian $\mathcal{L}$. Equations (6), (8), and (9) lead to

$$
\Phi_{i j}(q \mu, t)=\delta_{i j}\left[1-\left(\Omega_{q}^{s 2}+\Omega_{j}^{2}\right) t^{2} / 2\right]+O\left(t^{3}\right) .
$$

The correlators are normalized for $t=0$. The leading contribution to the transient does not introduce off-diagonal terms and it is given - independently of any interaction effects - by the two characteristic frequencies from Eq. (10). Because of reflection symmetry, the correlators $\Phi_{i j}(q \mu, t)$ do not depend on the sign of $\mu$. Exploiting $(A \mid B)=\left(B^{*} \mid A^{*}\right)$ as well as time-inversion symmetry one can show the following: the $\Phi_{i j}(q \mu, t)$ are real, even in $t$, and symmetric under interchanges of $i$ and $j$. For top-down symmetric molecules the correlators vanish if $i+j$ is odd.

\section{Equations of motion}

In this section two coupled equations of motion shall be derived. The first one relates the time derivative of the matrix $\Phi(t)$ of density correlators to the matrix $K(t)$ of momentum density correlations. The second one connects the time derivative of $K(t)$ with a matrix $M(t)$ of fluctuating-force correlations.

The Zwanzig-Mori formalism provides a procedure to formulate equations of motion for a set of distinguished variables. The coupling of these variables to the other variables 
of the systems is hidden in memory kernels, so that the equations of motion get the form of integrodifferential equations of the Volterra type $[2-4,42,46]$. Let us start by choosing as set of distinguished variables the density fluctuations $\varrho_{i}(q \mu)$ from Eq. (16b). Since $\left(\varrho_{i}(q \mu) \mid \mathcal{L} \varrho_{j}(q \mu)\right)=0$, the first equation of motion reads

$$
\partial_{t} \Phi_{i j}(q \mu, t)+\sum_{\bar{\imath}} \int_{0}^{t} d \bar{t} \Psi_{i \bar{\imath}}(q \mu, t-\bar{t}) \Phi_{\bar{\iota} j}(q \mu, \bar{t})=0,
$$

which has to be solved with the initial condition $\Phi_{i j}(q \mu, 0)$ $=\delta_{i j}$.

Let $\left.\mathcal{P}^{\prime}=\Sigma_{j} \mid \varrho_{j}(q \mu)\right)\left(\varrho_{j}(q \mu) \mid\right.$ denote the projector on the space of density fluctuations of wave vector $q$ and helicity $\mu$, and let $\mathcal{Q}^{\prime}=1-\mathcal{P}^{\prime}$ abbreviate the orthogonal complement. Since $\mathcal{P}^{\prime} \mathcal{L} \mathcal{P}^{\prime}=0$, one gets $\mathcal{Q}^{\prime} \mathcal{L} \varrho_{j}(q \mu)=\mathcal{L} \varrho_{j}(q \mu)$. The reduction of $\mathcal{L}$ onto the perpendicular space shall be denoted by $\mathcal{L}^{\prime}=\mathcal{Q}^{\prime} \mathcal{L} \mathcal{Q}^{\prime}$. The evolution with $\mathcal{L}^{\prime}$ shall be indicated by a prime: $A\left(t^{\prime}\right)=\exp \left(\mathrm{i} \mathcal{L}^{\prime} t\right) A$. Then the memory kernel in Eq. (20) is $\Psi_{i j}(q \mu, t)=\left(\mathcal{L} \varrho_{i}\left(q \mu, t^{\prime}\right) \mid \mathcal{L} \varrho_{j}(q \mu)\right)$. The density derivatives can be expressed as a sum of the two momentum density fluctuations $\sigma_{\alpha j}(q \nu)=\sigma_{\alpha j}^{\nu}\left(\vec{q}_{0}\right), \alpha=\mathrm{T}, \mathrm{R}$, according to Eq. (6). Thus $\Psi_{i j}$ can be written as a sum of four correlators of fluctuating momentum densities

$$
\Psi_{i j}(q \mu, t)=\sum_{\alpha \beta} \Omega_{\alpha i}(q) K_{\alpha i \beta j}(q \mu, t) \Omega_{\beta j}(q)
$$

where the matrix $K$ denotes

$$
\begin{aligned}
K_{\alpha i \beta j}(q \mu, t) & \\
= & {\left[\Omega_{\alpha i}(q) I_{\alpha}\right]^{-1}\left(\sigma_{\alpha i}\left(q \mu, t^{\prime}\right) \mid \sigma_{\beta j}(q \mu)\right)\left[\Omega_{\beta j}(q) I_{\beta}\right]^{-} }
\end{aligned}
$$

The pairs $(\alpha i),(\beta j)$ serve as matrix indices. For $i=0$, only the possibility $\alpha=\mathrm{T}$ occurs in the pair ( $\alpha i)$. Factors are split off in Eqs. (21) and (22) so, that Eqs. (9) and (10) imply

$$
K_{\alpha i \beta j}(q \mu, t=0)=\delta_{\alpha \beta} \delta_{i j} .
$$

The procedure shall be repeated using the momentum density fluctuations as variables. Since $\left(\sigma_{\alpha i}(q \mu) \mid \mathcal{L}^{\prime} \sigma_{\beta j}(q \mu)\right)=0$, one gets the second equation of motion,

$\partial_{t} K_{\alpha i \beta j}(q \mu, t)+\sum_{\bar{l}} \int_{0}^{t} d \bar{t} M_{\alpha i \bar{\alpha} \bar{l}}(q \mu, t-\bar{t}) K_{\bar{\alpha} \bar{l} \beta j}(q \mu, \bar{t})=0$,

which has to be solved with the initial condition (23).

Because of Eq. (9), the projector on the second relevant subspace reads $\left.\mathcal{P}^{\prime \prime}=\Sigma_{\alpha i} \mid \sigma_{\alpha i}(q \mu)\right)\left[\Omega_{\alpha i}(q) I_{\alpha}\right]^{-2}\left(\sigma_{\alpha i}(q \mu) \mid\right.$. The complement shall be denoted by $\mathcal{Q}^{\prime \prime}=1-\mathcal{P}^{\prime \prime}$. Since $\mathcal{P}^{\prime \prime} \mathcal{P}^{\prime}=0$ one gets $\mathcal{Q}=\mathcal{Q}^{\prime} \mathcal{Q}^{\prime \prime}=1-\mathcal{P}^{\prime}-\mathcal{P}^{\prime \prime}$. The fluctuations $f_{\alpha i}(q \mu)=\mathcal{L}^{\prime} \sigma_{\alpha i}(q \mu)=\mathcal{Q}^{\prime} \mathcal{L} \sigma_{\alpha i}(q \mu)$ have even timeinversion parity and therefore are orthogonal to the $\sigma_{\beta j}(q \mu): \mathcal{P}^{\prime \prime} \mathcal{L}^{\prime} \mathcal{P}^{\prime \prime}=0, \mathcal{Q}^{\prime \prime} \mathcal{L}^{\prime} \sigma_{\alpha i}(q \mu)=f_{\alpha i}(q \mu)$. Let us denote the new reduced Liouvillian by $\mathcal{L}^{\prime \prime}=\mathcal{Q}^{\prime \prime} \mathcal{L}^{\prime} \mathcal{Q}^{\prime \prime}=\mathcal{Q} \mathcal{L} \mathcal{Q}$ and indicate the corresponding time evolution by a double prime: $A\left(t^{\prime \prime}\right)=\exp \left(\mathrm{i} \mathcal{L}^{\prime \prime} t\right) A$. Thus the memory kernel $M$ can be written as the correlator of the fluctuating forces $F_{\beta j}(q \mu)=f_{\beta j}(q \mu) /\left[\Omega_{\beta j}^{2}(q) I_{\beta}\right]$ :

$$
\begin{gathered}
M_{\alpha i \beta j}(q \mu, t)=\Omega_{\alpha i}(q)\left(F_{\alpha i}\left(q \mu, t^{\prime \prime}\right) \mid F_{\beta j}(q \mu)\right) \Omega_{\beta j}(q), \\
F_{\beta j}(q \mu)=\left[\Omega_{\beta j}^{2}(q) I_{\beta}\right]^{-1} \mathcal{L} \sigma_{\beta j}^{\mu}\left(\vec{q}_{0}\right)-\varrho_{j}^{\mu}\left(\vec{q}_{0}\right) .
\end{gathered}
$$

The pair of equations (20), (24) is our generalization of the starting equation (1a) of the MCT for simple liquids. A new subtlety is the splitting of the current kernel $\Psi$ into four terms in Eq. (21). This splitting formalizes the idea that density fluctuations for $j \neq 0$ decay via parallel relaxation over two channels: decay due to translational and due to reorientational motion. The preceding equations are an exact reformulation of the problem; all difficulties are hidden in the kernel $M$. For top-down symmetric molecules $\Psi_{i j}(q \mu, t)$, $K_{\alpha i \beta j}(q \mu, t)$, and $M_{\alpha i \beta j}(q \mu, t)$ vanish if $i+j$ is odd. In this case the equations of motion consist of two uncoupled sets. One set deals with the matrices where both indices $i$ and $j$ are even, the other one deals with the matrices where both $i$ and $j$ are odd.

\section{A MODE-COUPLING THEORY}

If one performs a Markovian approximation for the relaxation kernel, $M(t)=\nu \delta(t)$, the equations of motion in Sec. II D reduce to master equations. Their solutions for the density correlator $\Phi(t)$ are sums of damped-oscillator functions. Such an approach would not deal properly with structuralrelaxation phenomena since stretching of relaxation is not obtained. The same holds if other elementary functions are used for $M(t)$ as, e.g., exponential decays [42]. Glassy relaxation of $\Phi$ is caused by that of the force correlations $M$. Nontrivial contributions to the forces $F$ are due to interactions of the molecule with atoms of the surroundings. Hence, structural-relaxation features of $M$ are connected with the slow relaxation of the molecule-atom separations. There is a self-consistency problem: the densities fluctuate slowly because the forces exhibit slow relaxation, and the forces relax sluggishly since the particle positions relax sluggishly. The theoretical problem is to express the force correlators $M(t)$ in terms of the density correlators $\Phi(t)$ so that glassy relaxation of the latter implies that of the former. In particular, the appearance of nonvanishing nonergodicity parameters for the density correlators must imply nonzero nonergodicity parameters for the force correlators [48-50]. In this section the preceding work on simple systems will be extended by approximating the kernel $M$ as a polynomial of the correlators. As a result closed equations of motion will be obtained for the functions $\Phi_{i j}(q \mu t)$, which contain the density correlators $\Phi_{q}(t)$ of the surroundings as input. A model for the molecule dynamics will be derived, whose subtleties are related to the appearance of nonlinearities and retardations.

\section{A. MCT equations of motion}

We restrict the discussion to the surroundings of $N$ identical spherical particles; generalizations to mixtures would be straightforward. Let $w\left(\vec{r}, \vec{e}, \vec{r}_{S}\right)$ denote the variable for finding the molecule at position $\vec{r}$ with orientation $\vec{e}$ and simul- 
taneously an atom of the surroundings at position $\vec{r}_{S}$. Fourier transformation of $w$ with respect to $\vec{r}$ and $\vec{r}_{S}$ and expansion of the $\vec{e}$ dependence in spherical harmonics yield $w$ as linear combination of

$$
A_{l}^{\kappa}(\vec{k} \vec{p})=\varrho_{l}^{\kappa}(\vec{k}) \varrho(\vec{p}) / \sqrt{N S(p)} .
$$

These pair modes are the simplest variables dealing with the molecule-atom pair configurations, and are expected to exhibit structural-relaxation dynamics. There are additional variables that can also contribute to the forces, such as the triple modes $\varrho_{l}^{\kappa}(\vec{k}) \varrho(\vec{p}) \varrho(\vec{q})$. The incorporation of these and more complicated modes would cause great difficulties. For example, it would be necessary to orthogonalize these complicated modes with respect to the $A_{l}^{\kappa}(\vec{k} \vec{p})$. To start such a procedure one would need three-particle and four-particle averages, about which not much information is available. Therefore, as a first step of our approximation procedure, all modes except the specified pair modes shall be ignored. This step is motivated by the intention to analyze the simplest version of a theory.

The second step is the factorization of averages of products into products of averages. This factorization approximation was introduced by Kawasaki $[51,52]$ in some different context. One can justify this approximation for several problems such as the determination of hydrodynamic long-time tails [30]. In the present context, unfortunately, one does not know the essence of the errors of the approximation, nor how to construct improvements. Making use of translational invariance, the approximation reads

$$
\left(A_{l^{\prime}}^{\kappa^{\prime}}\left(\vec{k}^{\prime} \vec{p}^{\prime}, t^{\prime \prime}\right) \mid A_{l}^{\kappa}(\vec{k} \vec{p})\right)=\delta_{k^{\prime} k} \delta_{p^{\prime} p} \Phi_{l^{\prime} l}^{\kappa^{\prime} \kappa}(\vec{k}, t) \Phi_{p}(t) .
$$

Here the tensor density fluctuation correlator entered via Eq. (15), and $\Phi_{q}(t)$ denotes the density correlator of the surrounding simple system, discussed in Sec. I. Equation (28) for $t=0$ implies the orthonormality of the pair modes.

Let $X$ and $Y$ denote two variables, which are perpendicular to the density and momentum density fluctuations: $\mathcal{Q} X$ $=X, \mathcal{Q} Y=Y$. Let us consider the correlator of these variables, where the reduced dynamics, as generated by $\mathcal{L}^{\prime \prime}$, is anticipated. We introduce the mode-coupling contribution to this correlator as the part in the subspace of the pair modes,

$$
\begin{aligned}
\left(X\left(t^{\prime \prime}\right) \mid Y\right)^{\mathrm{MC}}= & \sum_{\vec{k} p} \sum_{l^{\prime} l} \sum_{\kappa^{\prime} \kappa}\left(X \mid A_{l^{\prime}}^{\kappa^{\prime}}(\vec{k} \vec{p})\right) \Phi_{l^{\prime} l}^{\kappa^{\prime} \kappa}(\vec{k} t) \Phi_{p}(t) \\
& \times\left(A_{l}^{\kappa}(\vec{k} \vec{p}) \mid Y\right)
\end{aligned}
$$

The entire remainder of the correlators shall be denoted by $\left(X\left(t^{\prime \prime}\right) \mid Y\right)^{\mathrm{reg}}$ :

$$
\left(X\left(t^{\prime \prime}\right) \mid Y\right)=\left(X\left(t^{\prime \prime}\right) \mid Y\right)^{\mathrm{MC}}+\left(X\left(t^{\prime \prime}\right) \mid Y\right)^{\mathrm{reg}} .
$$

The third and last step of our derivation consists of the assumption that $\left(X\left(t^{\prime \prime}\right) \mid Y\right)^{\text {reg }}$ is regular in the sense that it deals with transient effects only. In particular, it is assumed that its long-time limit vanishes.
The preceding formulas shall be used with $X=F_{\alpha i}(q \mu)$, $Y=F_{\beta j}(q \mu)$ in order to treat the fluctuating-force kernel in Eq. (25):

$$
\begin{aligned}
M_{\alpha i \beta j}(q \mu, t)= & \Omega_{\alpha i}(q)\left[m_{\alpha i \beta j}(q \mu, t)\right. \\
& \left.+m_{\alpha i \beta j}^{\mathrm{reg}}(q \mu, t)\right] \Omega_{\beta j}(q) .
\end{aligned}
$$

The mode-coupling contribution $m(t)$ is a functional of the two correlators entering Eq. (29a)

$$
m_{\alpha i \beta j}(q \mu, t)=\mathcal{F}_{\alpha i \beta j q \mu}\left(\Phi_{l^{\prime} l}(k \nu, t) ; \Phi_{p}(t)\right) .
$$

The mode-coupling functional $\mathcal{F}$ is a polynomial of its variables

$$
\mathcal{F}_{\alpha i \beta j q \mu}\left(\tilde{f}_{l^{\prime} l}(k \nu) ; \tilde{f}_{p}\right)=\sum_{k p l^{\prime} l \nu} V_{q \mu k p l^{\prime} l \nu}^{\alpha i \beta j} \tilde{f}_{l^{\prime} l}(k \nu) \tilde{f}_{p} .
$$

Remembering Eqs. (16a) and (26) it is obvious that the coefficients $V$ are given by products of Wigner functions and overlaps of the pair modes from Eq. (27) with $\mathcal{L} \sigma$ and $\varrho$. Because of Eqs. (14) the latter are determined by geometry factors and by $S(p) c_{J}(p)$. Therefore, the $V$ are known functions depending smoothly on control parameters such as $n$ and $T$; details are noted in the Appendix.

The regular contribution to the fluctuating-force correlator $m^{\text {reg }}(t)$ is quite nontrivial. Imagine that there is no moleculeatom interaction at all, so that the $\Phi_{i j}(q \mu, t)$ describe freerotator motion. The resulting functions $C^{(j)}(t)$, Eq. (17), are quite complicated [42], and thus produced by complicated expressions for $m^{\mathrm{reg}}(t)$. Furthermore, within the normal liquid state there will be a coupling of the reorientational motion to the liquid's shear modes. This coupling was the basis of Debye's theory relating reorientational relaxation to the viscosity [42]. Such coupling also leads to some long-time power-law decay of $C^{(j)}(t)$ [53], yet another subtlety hidden in $m^{\text {reg }}(t)$. However, these and other normal liquid phenomena are irrelevant within that dynamical window, where structural relaxation dominates. In this window a Markov approximation for $m^{\text {reg }}(t)$ is sufficient.

The equations of motion (20)-(24) together with the mode-coupling-approximation results (30) are the desired closed set of equations for the molecule dynamics. These are the analog of the mode-coupling equations (1) for simple liquids. The approximations are constructed so that they deal with the translation and reorientation of the linear molecule in a regime, where glassy dynamics dominates. The dynamics of the surroundings enters the mode-coupling functional, Eq. (30b), via the density correlator $\Phi_{q}(t)$. Let us emphasize two features of our theory. Firstly, neither the inertia parameters of the molecule nor the temperature $T$ occur in the mode-coupling functional explicitly. Secondly, the interaction potential enters only indirectly via the structural quantities $n c_{J}(p)$ and $S(p)$. Therefore our theory can handle the strong repulsive forces that dominate the structure of molecular liquids.

The derived equations formalize the essential feedback mechanism of the MCT, which can lead to an ideal glass transition [48-50]. A white-noise approximation for the spectrum of the force kernel $M$ implies exponentially decay- 
ing contributions to the correlator $\Phi$, as mentioned above. Substituting this into Eqs. (30), one gets a low-frequency peak in the spectrum of the kernel. A white-noise approximation for the spectrum of $M$ is therefore inadequate; rather the low-frequency force spectrum is enhanced since the zerofrequency spectrum of the pair modes $A_{l}^{\kappa}(\vec{k} \vec{p})$ is enhanced. Such a peak in the kernel spectrum produces a corresponding peak in the correlator spectrum via the equations of motion (20)-(24) as first discussed by Mountain within Maxwell's theory of viscoelasticity [54]. Substituting this back into Eqs. (30), the quasielastic peak in the force spectrum gets enhanced further and this leads to a further enhancement of the corresponding quasielastic spectrum for the density fluctuations, etc. There are two possibilities for a stabilization of this feedback mechanism. Either there appear nonLorentzian quasielastic spectra for the kernel and the correlator, and this is the essence of the MCT for the $\alpha$ relaxation within the liquid, or the width of the Mountain peak collapses to zero, and this is the essence of the MCT for the ideal glass state. The area of the degenerate Mountain peak is the nonergodicity parameter of the correlator under discussion.

Mode coupling is achieved via the overlaps from Eq. (14a). For top-down symmetric molecules, variables of different parity do not couple. In this case, the complete MCT problem splits into two independent ones. The modecoupling expressions (30) do not introduce coupling between the two sets specified at the end of Sec. II D.

\section{B. The glass form factors}

Let us denote the long-time limits of the density correlators by

$$
f_{i j}(q \mu)=\lim _{t \rightarrow \infty} \Phi_{i j}(q \mu, t)
$$

These nonergodicity parameters or glass form factors-if they are nonzero-quantify the spontaneous arrest of the molecule in its environment. They are the Lamb-Mössbauer factors that determine via (18) the intensity of the strictly elastic scattering of test particles, like neutrons, from the molecule. The Edwards-Anderson parameters $f_{j}$, which were defined in Eq. (4), are obtained via Eq. (17) as longwavelength limits

$$
f_{i j}(q \rightarrow 0, \mu)=\delta_{i j} f_{j}, \quad|\mu| \leqslant \min (i, j) .
$$

The mode-coupling formulas (30) establish a representation of the nonergodicity parameter of the fluctuating forces, to be quantified by $g_{\alpha i \beta j}(q \mu)$ via $M_{\alpha i \beta j}(q \mu, t \rightarrow \infty)$ $=\Omega_{\alpha i}(q) g_{\alpha i \beta j}(q \mu) \Omega_{\beta j}(q)$, in terms of those for the molecule and for the surroundings, Eqs. (2), (31a),

$$
g_{\alpha i \beta j}(q \mu)=\mathcal{F}_{\alpha i \beta j q \mu}\left(f_{l^{\prime} l}(k \nu) ; f_{p}\right)
$$

To proceed one has to derive a further relation between $g$ and $f$ via the equations of motion from Sec. II D. This is done most efficiently [48-50] using Laplace transformations, for which we apply the convention $F(z)$ $=\mathrm{i} \int_{0}^{\infty} F(t) \exp (\mathrm{i} z t) d t, \operatorname{Im} z \geqslant 0$. A nonzero long-time limit, $F(t \rightarrow \infty)=F_{\infty}$, is equivalent to the existence of a nonergod- icity pole at zero frequency: $-z F(z) \rightarrow F_{\infty}$ for $z \rightarrow 0$. The first Zwanzig-Mori equation (20) together with its initial condition is equivalent to

$$
-\sum_{\bar{l}}\left[z \delta_{i \bar{\imath}}+\Psi_{i \bar{\imath}}(q \mu, z)\right] \Phi_{{ }_{l} j}(q \mu, z)=\delta_{i j}
$$

and the second one, Eq. (24), reads

$$
-\sum_{\bar{\alpha} \bar{l}}\left[z \delta_{i \bar{l}} \delta_{\alpha \bar{\alpha}}+M_{\alpha i \bar{\alpha} \bar{l}}(q \mu, z)\right] K_{\bar{\alpha} \bar{l} \beta j}(q \mu, z)=\delta_{i j} \delta_{\alpha \beta}
$$

The zero-frequency limit of Eq. (33a) yields

$$
\sum_{\bar{l}}\left[\delta_{i \bar{\imath}}+J_{i}-(q \mu)\right] f_{\bar{l}}(q \mu)=\delta_{i j}
$$

This formula relates the glass form factors with the limit of the current correlators: $J_{i j}(q \mu)=\lim _{z \rightarrow 0} \Psi_{i j}(q \mu, z) / z$. Because of Eq. (33b), the $J_{i j}$ are combinations of the corresponding asymptotic values $\lim _{z \rightarrow 0} \Omega_{\alpha i}(q) K_{\alpha i \beta j}(q \mu, z) \Omega_{\beta j}(q) / z=I_{\alpha i \beta j}(q \mu)$,

$$
J_{i j}(q \mu)=\sum_{\alpha \beta} I_{\alpha i \beta j}(q \mu) .
$$

The zero-frequency limit of Eq. (33b) together with Eq. (30a) identifies the matrix $I$ as the inverse of the matrix $g$ :

$$
\sum_{\bar{\alpha}} g_{\alpha i \bar{\alpha}}-(q \mu) I_{\bar{\alpha}}{ }_{l}{ }_{\beta j}(q \mu)=\delta_{\alpha \beta} \delta_{i j}
$$

The formulas (32) and (34) are the desired equations for the form factors. These coupled nonlinear equations have to be complemented by the equation for the $f_{q}$, cited in Sec. I, since these functions enter Eq. (32) as input. It is known from the earlier literature that implicit equations of the kind formulated above can exhibit two generic types of ergodicto-nonergodic transitions at a critical value $x_{c}$ of some control parameter $x$, such as $n$ or $1 / T$. The form factors may rise continuously proportional to $\left(x-x_{c}\right)$ [48-50,55-57] (type- $A$ transitions), or they may jump to nonzero values $f^{c}[28,58]$ (type- $B$ transitions). Below it will be demonstrated that both types of transitions can occur in our theory.

\section{Further approximations}

The appearance of matrix correlators instead of autocorrelation functions implies a considerable increase in the efforts to solve the derived equations. Matrix generalizations of the original MCT equations have been derived and solved before for the studies of mixtures of spherical molecules $[40,59,60]$. It turned out that the generalizations merely caused technical problems, which did not lead to new qualitative features. Therefore we suggest to start studies of molecular liquids with the additional approximation, which ignores off-diagonal force correlators: $M_{\alpha i \beta j}(q \mu, t)$ $\simeq \delta_{\alpha \beta} \delta_{i j} M_{\alpha}(q j \mu, t)$. This approximation implies diagonality for the current and density correlators: $K_{\alpha i \beta j}(q \mu, t)$ $=\delta_{\alpha \beta} \delta_{i j} K_{\alpha}(q j \mu, t), \Phi_{i j}(q \mu, t)=\delta_{i j} \Phi(q j \mu, t)$. Within this 
diagonal approximation, also index $j$ enters the theory as a parameter like $q$ and $\mu$. The equations of motion of Sec. II D simplify to

$$
\begin{gathered}
\partial_{t} \Phi(q j \mu, t)+\int_{0}^{t} d \bar{t} \Psi(q j \mu, t-\bar{t}) \Phi(q j \mu, \bar{t})=0, \\
\Psi(q j \mu, t)=\Omega_{q}^{s 2} K_{\mathrm{T}}(q j \mu, t)+\Omega_{j}^{2} K_{\mathrm{R}}(q j \mu, t), \\
\partial_{t} K_{\alpha}(q j \mu, t)+\int_{0}^{t} d \bar{t} M_{\alpha}(q j \mu, t-\bar{t}) K_{\alpha}(q j \mu, \bar{t})=0 .
\end{gathered}
$$

The mode-coupling formulas closing these equations follow from Eqs. (30):

$$
\begin{gathered}
M_{\alpha}(q j \mu, t)=\Omega_{\alpha j}(q)^{2}\left[m_{\alpha}(q j \mu, t)+m_{\alpha}^{\mathrm{reg}}(q j \mu, t)\right], \\
m_{\alpha}(q j \mu, t)=\mathcal{F}_{\alpha q j \mu}\left(\Phi(k l \nu, t) ; \Phi_{p}(t)\right), \\
\mathcal{F}_{\alpha q j \mu}\left(\widetilde{f}(k l \nu) ; \widetilde{f_{p}}\right)=\sum_{k p l \nu} V_{\alpha q j \mu}(k p l \nu) \widetilde{f}(k l \nu) \widetilde{f}_{p} .
\end{gathered}
$$

The $V_{\alpha q j \mu}(k p l \nu)=V_{q \mu k p l l \nu}^{\alpha j \alpha j}$ are non-negative numbers, and therefore our mode-coupling functional has the same general properties as the one for the simple-liquid theory. The equations for the form factors $f(q j \mu)=\Phi(q j \mu, t \rightarrow \infty)$ are obtained from Sec. III B

$$
\begin{gathered}
f(q j \mu)=\frac{1}{1+g_{\mathrm{T}}(q j \mu)^{-1}+g_{\mathrm{R}}(q j \mu)^{-1}}, \\
g_{\alpha}(q j \mu)=\mathcal{F}_{\alpha q j \mu}\left(f(k l \nu) ; f_{p}\right) .
\end{gathered}
$$

For $j=0$, the density correlator specializes to the selfcorrelation function of the molecule density: $\Phi(q 00, t)$ $=\Phi_{q}^{s}(t)$. In this case Eq. (35b) simplifies to $\Psi(q 00, t)$ $=\left(q v_{\mathrm{T}}\right)^{2} K_{\mathrm{T}}(q 00, t)$ so that the equations of motion can be combined to

$$
\partial_{t}^{2} \Phi_{q}^{s}(t)+\left(q v_{\mathrm{T}}\right)^{2} \Phi_{q}^{s}(t)+\int_{0}^{t} d \bar{t} M_{q}^{s}(t-\bar{t}) \partial_{\bar{t}} \Phi_{q}^{s}(\bar{t})=0 .
$$

This result is the analog of Eq. (1a) and has been considered before for simple liquids [28]; but here a coupling to the rotational degrees of freedom is hidden in the formula for $M_{q}^{s}(t)=\left(q v_{\mathrm{T}}\right)^{2}\left[m_{q}^{s}(t)+m_{q}^{s, \text { reg }}(t)\right]$ via the kernel $m_{q}^{s}(t)$ $=m_{\mathrm{T}}(q 00, t)$, Eqs. (36).

For $q=0$ and $j \neq 0$, the density correlators specialize to the rotator functions because of Eq. (17). In this case Eq. (35b) simplifies to $\Psi(0 j \mu, t)=v_{\mathrm{R}}^{2} j(j+1) K_{\mathrm{R}}(0 j 0, t)$. Let us note the result in the frequency domain

$$
C^{j}(z)=-1 /\left\{z-v_{\mathrm{R}}^{2} j(j+1) /\left[z+M_{j}(z)\right]\right\} .
$$

This equation reproduces the representation of $\Phi_{j}(z)$ as a two-step continued fraction [42]. The subtleties of the dynamics, in particular the couplings to the translation, are hidden in the kernel $M_{j}(z)=v_{\mathrm{R}}^{2} j(j+1)\left[m_{j}^{\mathrm{R}}(z)+m_{j}^{\mathrm{R}, \text { reg }}(z)\right]$ via $m_{j}^{\mathrm{R}}(t)=m_{\mathrm{R}}(0 j 0, t)$, Eqs. (36).
There is no experimental evidence indicating a connection between structural relaxation and appearance of long-range spatial correlations. The available experimental evidence suggests that there is no singular small- $q$ behavior of the structural quantities $n c_{J}(q), S(q)$; the relaxation kernels $M$ are expected to vary regularly in leading order for small $q$. If regular structure factors are used as input, our modecoupling formulas for $M$ reproduce such regularity for the leading-order small- $q$ variations. Long-wavelength correlations can therefore in leading order be handled correctly by ignoring the $q$ dependence of the kernels. This suggests a generalized hydrodynamic approximation as a further simplification of our results: $M_{\alpha}(q j \mu, t) \simeq M_{\alpha}(0 j \mu, t)$. The approximation implies independence of the correlators of the helicity $\mu$, because of rotational symmetry. Equations (35) for $\Phi_{j q}(t)=\Phi(q j \mu, t)$ simplify to

$$
\Phi_{j q}(z)=-1 /\left[z-\frac{v_{\mathrm{T}}^{2} q^{2}}{z+M_{j \mathrm{~T}}(z)}-\frac{v_{\mathrm{R}}^{2} j(j+1)}{z+M_{j \mathrm{R}}(z)}\right],
$$

where $M_{j \alpha}(z)$ is the $q \rightarrow 0$ limit of the $M_{\alpha}(q j \mu, z)$ from Eq. (36a). The glass form factors are specified by length parameters $r_{j}$ and by constants $s_{j}$ :

$$
f_{j q}=1 /\left[1+\left(q r_{j}\right)^{2}+s_{j}^{2}\right] .
$$

Here $1 / r_{j}^{2}$ and $1 / s_{j}^{2}$ are $q=0$ limits of $q^{2} \mathcal{F}_{T q j \mu}\left(f_{l k \nu} ; f_{p}\right)$ and $\mathcal{F}_{R q j \mu}\left(f_{l k \nu} ; f_{p}\right)$, respectively.

\section{RESULTS AND DISCUSSIONS}

\section{A. Glass form factors for a hard dumbbell in a hard-sphere glass}

In this section some specific implications of our theory shall be considered and quantitative results shall be presented for a molecule in a system of hard spheres with diameter $d$. The equilibrium structure of the surroundings is specified by a single control parameter, say the packing fraction $\varphi=\pi d^{3} n / 6$. The structure factor $S(q)$ is calculated within the Percus-Yevick theory [3]. From Eqs. (1) one gets the form factors $f_{q}$ of the hard-sphere glass and finds a liquid-glass transition at the critical packing fraction $\varphi_{c}$ $=0.516$ [28]. If $\varphi$ decreases to $\varphi_{c}$, the glass form factor decreases to its critical value $f_{q}^{c}>0$ :

$$
f_{q}=f_{q}^{c}+h_{q} g(\sigma)+O_{q}(\sigma), \quad \varphi \geqslant \varphi_{c} .
$$

Here $h_{q}>0$ denotes the critical amplitude, and $g(\sigma)$ $=\sqrt{\sigma /(1-\lambda)}$ contains the square-root singularity characteristic for a fold bifurcation. The number $\lambda=0.735$ is called the exponent parameter and $\sigma \approx 1.5\left(\varphi-\varphi_{c}\right) / \varphi_{c}$ denotes the separation parameter. A detailed discussion of Eq. (42) and representative figures for $S(q), f_{q}^{c}$, and $h_{q}$, and $O_{q}$ can be found in Ref. [32]. The numerical work is done for dumbbell molecules consisting of two fused hard spheres with diameters $d_{1}$ and $d_{2}$. The distance between the centers $\zeta d$ is specified by the elongation parameter $\zeta$. The unit of length shall be chosen so that $d=1$. For symmetric dumbbells $d_{1}$ $=d_{2}$, there is the additional top-down symmetry. The coupling functions $n c_{J}(q)$ are also calculated within the PercusYevick theory [61]. 


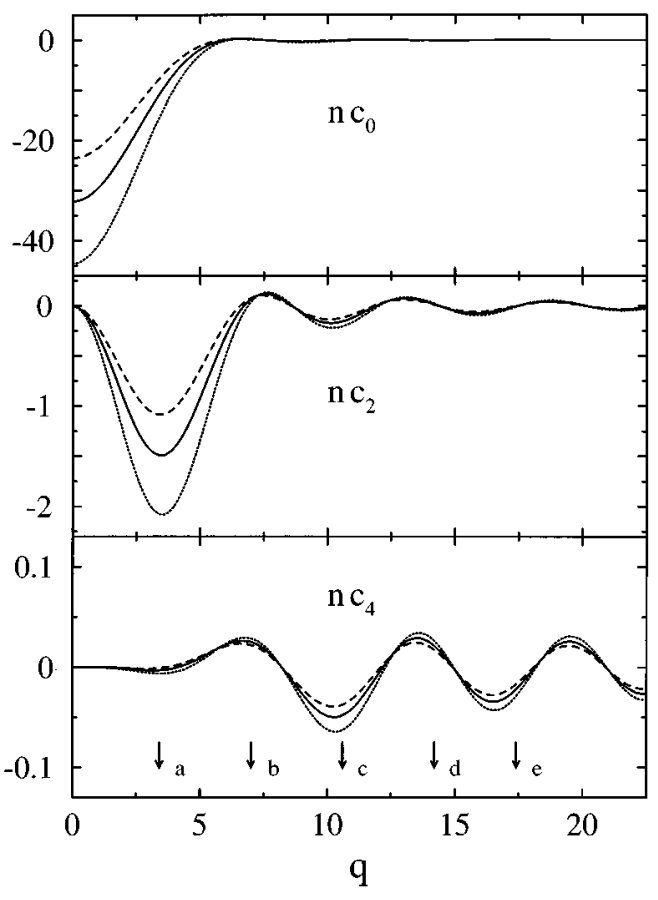

FIG. 1. Effective interaction potentials $n c_{J}(q)$ as defined by Eq. (11) for a dumbbell with $d_{1}=d_{2}=d$ and elongation parameter $\zeta$ $=0.4$ for $J=0,2$ and 4 . The packing fractions of the hard-sphere surroundings are $\varphi=0.49$ (dashed), $\varphi=0.52$ (solid), and $\varphi=0.55$ (dotted). The arrows indicate the $q$ values 3.4 (a), 7.0 (b), 10.6 (c), 14.2 (d), and 17.4 (e). Notice that the vertical scale of successive panels differs by a factor 20 .

Figure 1 exhibits a representative set of effective potentials $n c_{J}(q)$ for $\varphi$ near $\varphi_{c}$. The strong short-range repulsion between dumbbell and the neighboring hard spheres causes $c_{J}(q)$ to be negative for $q d \ll 2 \pi$. The rapid variation of the potential as a function of distance leads to rapid variations for the pair correlation as a function of angle and distance. This yields oscillations of $c_{J}(q)$ that extend up to rather large values of the wave vector $q$. The effective potential is a smooth function except for some isolated lines [61], and therefore the coefficients of expansions in spherical harmonics decrease with increasing $J$. The relevant coupling coefficients of our theory, $n c_{J}(q)$, become small if $J$ becomes large, in particular $n c_{J}(q)=O\left(q^{J}\right)$ for $q \rightarrow 0$. With increasing $\varphi$ the $\left|n c_{J}(q)\right|$ increase smoothly.

If the surroundings are a liquid, i.e., if $f_{q}=0$, the longtime limits $g_{\alpha i \beta j}(q \mu)$ of the fluctuating-force correlator, Eqs. (30c) and (32), vanish, and therefore the glass form factors $f_{i j}(q \mu)$ are zero as well. In this case the cage around the molecule has a finite lifetime and arrest is not possible. Let us therefore restrict the discussion to the glass state $\varphi$ $\geqslant \varphi_{c}$, concentrate on molecules with $d_{1}=d_{2}=d$, and use the diagonal approximation. Equation (37) is solved by the iteration $\quad f^{(n+1)}(q j \mu)=1 /\left[1+g_{\mathrm{T}}^{(n)}(q j \mu)^{-1}+g_{\mathrm{R}}^{(n)}(q j \mu)^{-1}\right]$, $g_{\alpha}^{(n)}(q j \mu)=\mathcal{F}_{\alpha q j \mu}\left(f^{(n)}(k l \nu) ; f_{p}\right), \quad f^{(0)}(q j \mu)=1, \quad n$ $=0,1, \ldots$. Figure 2 exhibits the resulting form factors at the critical point, $\varphi=\varphi_{c}$, for helicity $\mu=0$ and three representative values for the elongation parameter $\zeta$. Figure 3 shows analogous results for all helicities for $\zeta=0.6$. For $q$ $=0$ there is no dependence on $\mu$, Eq. (31b), and for large $q$ the nonlinear mode-coupling effects force the functions to

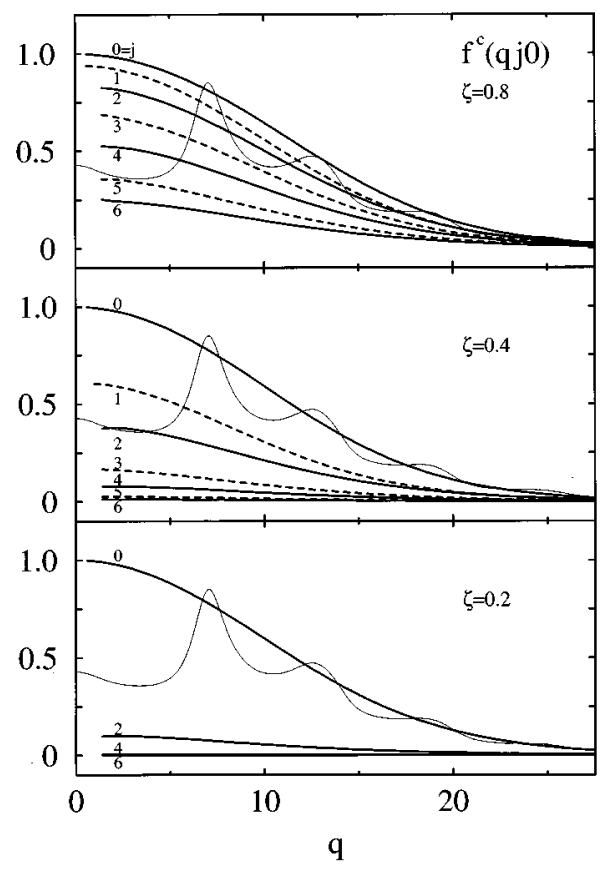

FIG. 2. Glass form factors $f^{c}(q j 0)$ at the critical packing fraction $\varphi_{c}=0.516$ for $d_{1}=d_{2}=d$ and the three values for the elongation parameters $\zeta$. The labels at the curves indicate the value of $j$ $=0, \ldots, 6$. The thin lines show the critical form factor $f_{q}^{c}$ of the hard-sphere system.

approach zero roughly at the same wave vector. Therefore there is only a weak $\mu$ dependence of the form factors. For the tagged-particle motion, particle-number conservation implies $f(q=0,00)=1$. There is no analogous conservation law for the other values $j \neq 0$, and therefore $f(q=0, j \mu)=f_{j}<1$. The $f(q j \mu)$ can be approximated well by Gaussians $f^{c}(q j 0)$ $\propto \exp -\left(q a_{j}\right)^{2}$, as demonstrated in Fig. 4. This was noticed

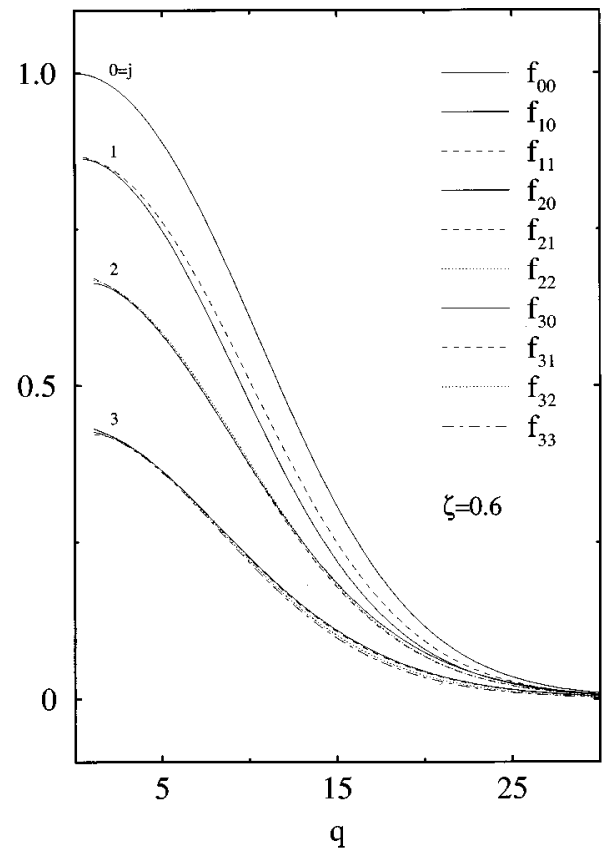

FIG. 3. Critical glass form factors $f_{j \mu}=f^{c}(q j \mu)$ for $d_{1}=d_{2}$ $=d$ and $\zeta=0.6$. 


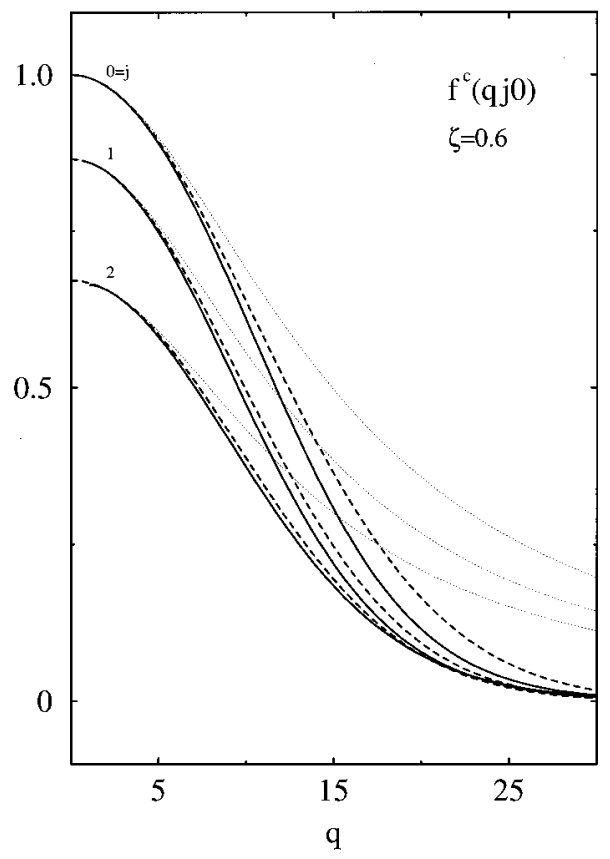

FIG. 4. Critical glass form factors $f^{c}(q j 0)=f_{j q}$ for $d_{1}=d_{2}=d$ and $\zeta=0.6$ for $j=0,1$, and 2 (full lines). The dotted lines show the approximation by the generalized hydrodynamics result $f_{j q}=1 /[1$ $\left.+\left(q r_{j}\right)^{2}+s_{j}^{2}\right]$ with $r_{j}$ and $s_{j}$ adjusted to match the small- $q$ behavior. The dashed lines are fits by Gaussians $f_{j q}=\exp \left[-\left(q a_{j}\right)^{2}\right] /$ $\left[1+s_{j}^{2}\right], a_{j}^{2}=r_{j}^{2} /\left[1+s_{j}^{2}\right]$.

before for the localization of a hard sphere in a hard-sphere glass [28]. The description of $f^{c}(q j 0)$ by the generalizedhydrodynamics formula (41) works reasonably well for wave vectors up to the position of the structure-factor peak near $q \sim 7$, but it leads to a severe overestimate of the form factors for $q \geqslant 10$.

The function $f(q 00)=f_{q}^{s}$ describes the localization of the molecule's center in the glass matrix. This function exhibits only a very weak variation with $\zeta$ as shown in the upper panel of Fig. 5. For $\zeta=0$ the dumbbell degenerates to a sphere, and in this case $f(q j \mu)=0$ for $j \neq 0$. The larger $\zeta$ the more the molecules differ from a spherical one and thus the $j \neq 0$ form factors increase appreciably with increasing $\zeta$, as shown in Figs. 2 and 5.

Arrest is less pronounced for strongly wiggled angular distributions than for smooth ones, since the neighboring spheres cannot resolve the variations of $Y_{j}^{\nu}(\vec{e})$ if $j$ is too large. Therefore one expects that the form factors $f(q j \mu)$ decrease with increasing $j$, as demonstrated in Figs. 2 and 3 for $\zeta \geqslant 0.4$. However, for top-down symmetric molecules it can happen that all $f_{i j}(q \mu)=0$ for odd $i, j$, while the form factors for even $i, j$ are nonzero. This is shown for $\zeta=0.2$ in Fig. 2. There is a critical value $\zeta_{c}$ so that $f_{i j}^{c}(q \mu)=0$ for odd $i, j$ and $\zeta \leqslant \zeta_{c}$. The odd- $j$ form factors are nonzero for $\zeta$ $>\zeta_{c}$ and they approach zero continuously if $\zeta$ decreases towards $\zeta_{c}$ as shown in Fig. 5. For a model with $d_{1}=d_{2}=d$, one finds $\zeta_{c}=0.296$ for $\varphi=\varphi_{c}$. Thus, for sufficiently small positive $\zeta-\zeta_{c}$ it can happen that, e.g., $f^{c}(q, 20)>f^{c}(q, 10)$ $>0$.

Formulas (32) and (34) define a set of implicit equations for the $f_{i j}(q \mu)$, which has the solution $f_{i j}^{c}(q \mu)$ for $\varphi=\varphi_{c}$

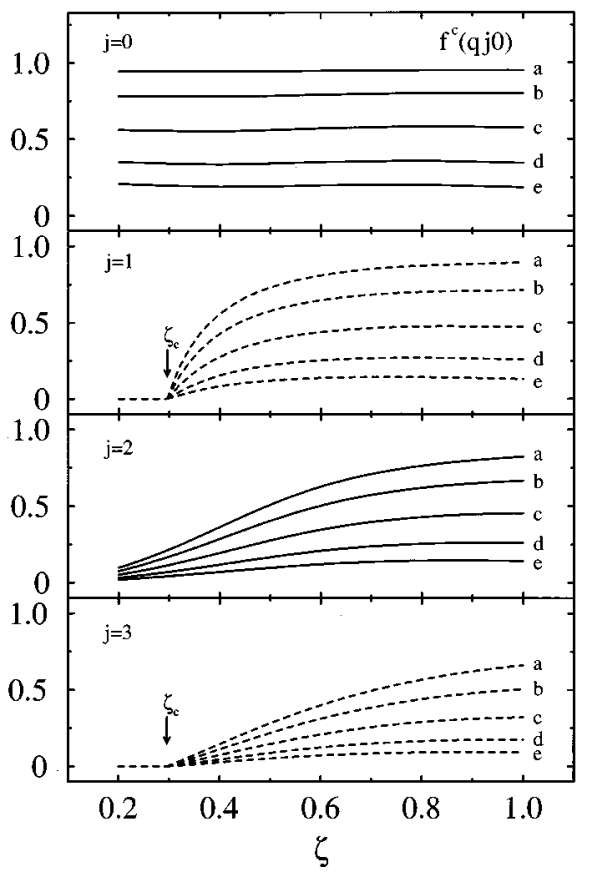

FIG. 5. Critical glass form factors $f^{c}(q j \mu)$ for helicity $\mu=0$ and $j=0, \ldots, 3$ as a function of the elongation parameter $\zeta$ for $d_{1}$ $=d_{2}=d$ and $q=3.4$ (a), 7.0 (b), 10.6 (c), 14.2 (d), and 17.4 (e). The arrows mark the critical value $\zeta_{c}=0.296$ for a type- $A$ transition.

and $f_{q}=f_{q}^{c}$. The equations depend smoothly on $\varphi$. But there appears a singular $\sqrt{\varphi-\varphi_{c}}$ dependence via Eq. (42) for the Debye-Waller factors $f_{q}$. Hence, one concludes from the implicit-function theorem for generic choices of $d_{1}, d_{2}, \zeta$,

$$
f_{i j}(q \mu)=f_{i j}^{c}(q \mu)+h_{i j}(q \mu) g(\sigma)+O_{i j q}(\sigma), \quad \varphi \geqslant \varphi_{c} .
$$

One concludes that the nonergodicity parameters for the molecule exhibit the same $\sqrt{\varphi-\varphi_{c}}$ singularity as the DebyeWaller factors. This is demonstrated in Figs. 6 and 7 for two values of $\zeta$; notice that for $\zeta<\zeta_{c}$ the critical amplitudes $h(q j 0)$ vanish for odd $j$. Figure 8 exhibits representative results for $h$ versus $q$ curves. For large wave vectors the $h_{i j}(q \mu)$ have to approach zero since $f_{i j}(q \mu)$ vanishes in the limit $q \rightarrow \infty$. Since $f_{00}(q \rightarrow 0,0)=1$ for all $\varphi \geqslant \varphi_{c}$, the amplitude $h_{00}(q 0)$ has to vanish in the long-wavelength limit. Hence, $h_{00}(q 0)$ is bell shaped and it exhibits a maximum for a wave vector that is about twice the position of the $S(q)$ maximum, as was found before for a spherical molecule [62]. From Eq. (31b) one gets the result for the square-root anomaly of the Edwards-Anderson parameters $f_{j}=f_{j}^{c}$ $+h_{j} g(\sigma)+O(\sigma)$. For example, one finds for $\zeta=0.4: f_{1}^{c}$ $\approx 0.61, f_{2}^{c} \approx 0.39, h_{1} \approx 0.98, h_{2} \approx 0.57$. This means that $f_{1}$ increases more strongly with $\varphi-\varphi_{c}$ than $f_{2}$ as demonstrated in Fig. 6. The findings $f_{1}^{c}>f_{2}^{c}$ and $h_{1}>h_{2}$ for $\zeta=0.4$ are in qualitative agreement with molecular dynamics results for OTP $[17,63]$.

\section{B. The factorization theorem}

The essential point in the derivation of the asymptotic laws for the MCT bifurcation dynamics is the following observation: for small $\left|\varphi-\varphi_{c}\right|$, there appears an intermediate- 


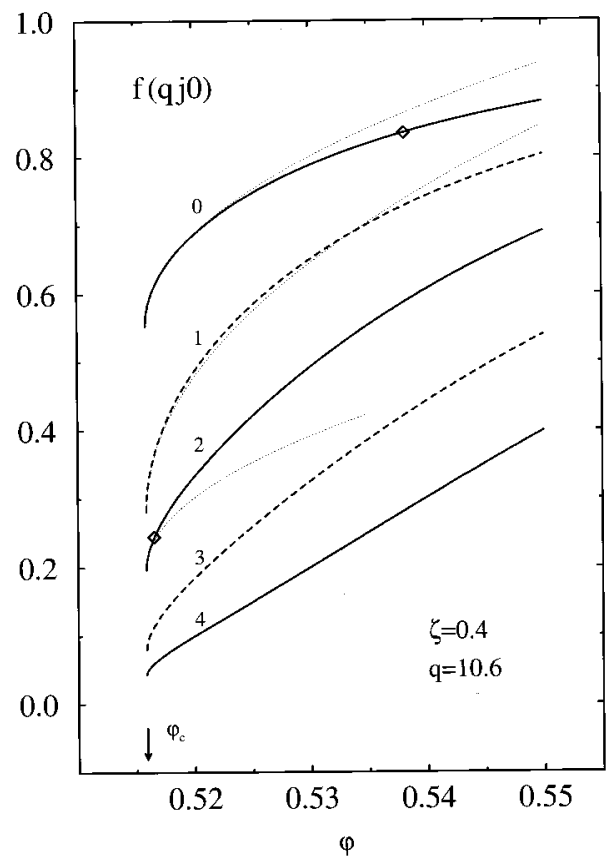

FIG. 6. Form factors $f(q j 0)$ for $j=0, \ldots, 4$ and $q=10.6$ as a function of the packing fraction $\varphi$ for $\zeta=0.4$ and $d_{1}=d_{2}=d$. The labels at the curves denote the value $j$. The arrow indicates the glass transition point $\varphi_{c}=0.516$. The dotted lines are the asymptotes $f(q j 0)=f^{c}(q j 0)+h(q j 0) \sqrt{\sigma /(1-\lambda)}$ for $j=0,1$, and 2 . The diamonds mark for $j=0,2$ the values of $\varphi$ where $f(q j 0)$ differs from the asymptote by $10 \%$ of $f(q j 0)-f^{c}(q j 0)$.

time window, referred to as the $\beta$-relaxation region, where the correlators $\Phi_{q}(t)$ are close to the critical value $f_{q}^{c}$ of the nonergodicity parameter. The equations of motion can be simplified by using $\delta \Phi_{q}(t)=\Phi_{q}(t)-f_{q}^{c}$ as a small quantity for expansions. In leading order one obtains the factorization theorem

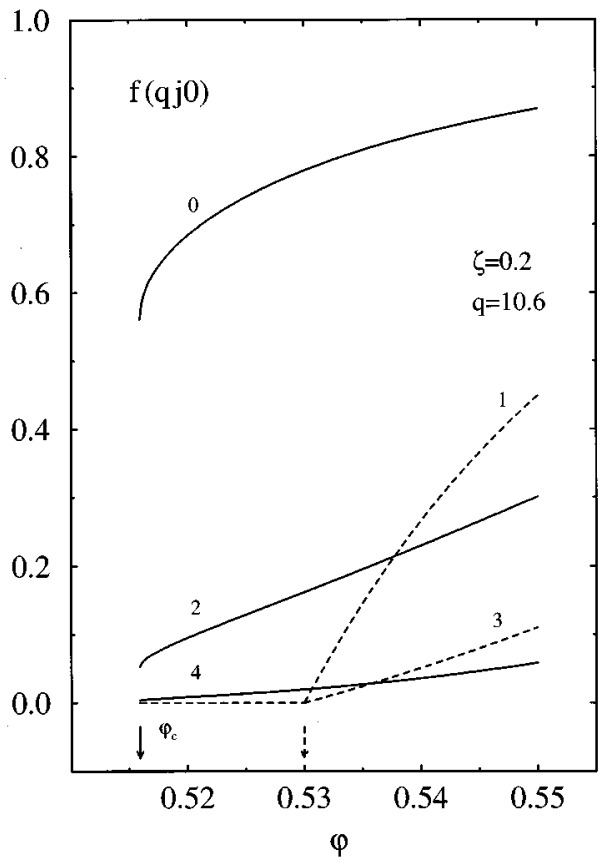

FIG. 7. Similar results as in Fig. 6 but for $\zeta=0.2$. The dashed arrow marks the value $\varphi=0.53$ for a type- $A$ transition point.

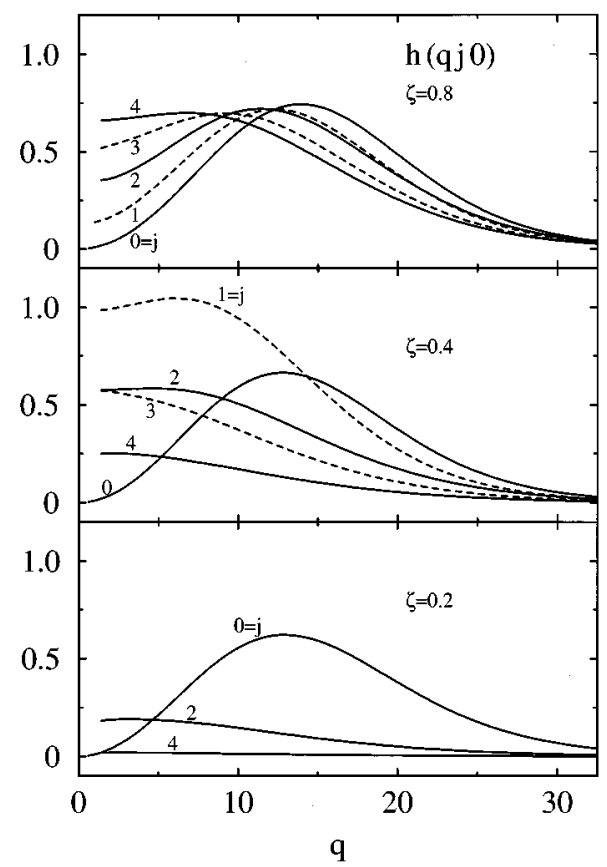

FIG. 8. Critical amplitudes $h(q j 0)$ for helicity zero as function of wave vector $q$ for $d_{1}=d_{2}=d$ and three values of the elongation $\zeta$. The labels at the curves denote the value of $j$.

$$
\Phi_{q}(t)-f_{q}^{c}=h_{q} G(t) .
$$

The deviation $\delta \Phi_{q}(t)$ of the correlator from the plateau $f_{q}^{c}$ factorizes into the critical amplitude $h_{q}$ from Eq. (42) and into a function $G(t)$, which is called the $\beta$ correlator. The latter exhibits the complete time dependence and also the sensitive dependence on control parameters via the separation parameter $\sigma: G(t)=g\left(t / t_{0}, \sigma\right)$. Here $t_{0}$ is some time scale determined by the transient dynamics. The function $g$ of the two variables $t / t_{0}$ and $\sigma$ is completely fixed by the exponent parameter $\lambda$. The function $G(t)$ quantifies a scaling law; in particular, it deals with the crossover between two fractal power-law decays. Equation (42) is a special implication of Eq. (44a), since for $\sigma>0$ one gets $G(t \rightarrow \infty)$ $=g(\sigma)$. All universality features of the MCT scenario for the evolution of structural relaxation are based on Eq. (44a) and the well-understood properties of $G(t)$ [33]. A detailed demonstration of the result (44a) and a discussion of its leading corrections can be found in Ref. [32].

The proof of Eq. (44a) can be extended to a treatment of other correlators such as the tagged-particle-correlation function $\Phi_{q}^{s}(t)$, the mean square displacements, or transversalshear correlations [64]. For our case one can adopt the known proofs to derive the generalization of Eq. (43) to the leading-order $\beta$-relaxation formula

$$
\Phi_{i j}(q \mu, t)-f_{i j}^{c}(q \mu)=h_{i j}(q \mu) G(t) .
$$

The molecule probes with amplitude $h_{i j}(q \mu)$ the $\beta$ relaxation dynamics of the surroundings as described by the function $G(t)$.

The identification of universality features for structural relaxation via the function $G(t)$ is an important outcome of MCT and therefore some digression might be in order. Let $Q_{i}, i=1,2, \ldots$, denote a complete set of single-particle 
variables. For the model studied in this paper, these are the molecule density variables $\varrho_{j}^{\nu}(\vec{q})$ and the density fluctuations of the molecule's surroundings $\varrho(\vec{k})$; the index $i$ is meant to abbreviate the set of $(\vec{k}, \vec{q}, j \nu)$. Let $\left(Q_{i_{1}} \cdots Q_{i_{L}}\right)$, $L=1,2, \ldots$, denote $L$-particle variables, i.e., the set of orthonormalized products of $L$ factors $Q_{i}$ [30]. These variables can exhibit structural arrest. Therefore, one writes for the correlator $\Phi_{X Y}(t)=\left\langle X(t)^{*} Y\right\rangle$ of two variables $X$ and $Y$

$$
\Phi_{X Y}(t)=\Phi_{X Y}^{\mathrm{reg}}(t)+\Phi_{X Y}^{\mathrm{MC}}(t) .
$$

Here $\Phi_{X Y}^{\mathrm{reg}}(t)$ denotes the contribution due to all those modes, which cannot arrest. It does not exhibit leading-order structural-relaxation effects. The second contribution reads

$$
\begin{aligned}
& \Sigma_{L} \Sigma_{i j}\left\langle X^{*}\left(Q_{i_{1}} \cdots Q_{i_{L}}\right)\right\rangle\left\langle\left(Q_{i_{1}} \cdots Q_{i_{L}}\right)(t)^{*}\left(Q_{j_{1}} \cdots Q_{j_{L}}\right)\right\rangle \\
& \quad \times\left\langle\left(Q_{j_{1}} \cdots Q_{j_{L}}\right) * Y .\right.
\end{aligned}
$$

It is approximated by products of correlators and yields the mode-coupling term

$$
\Phi_{X Y}^{\mathrm{MC}}(t)=\mathcal{F}_{X Y}\left(\Phi_{i j}(t)\right)
$$

The functional $\mathcal{F}_{X Y}$ is a polynomial in the correlators $\Phi_{i j}(t)=\left\langle Q_{i}(t)^{*} Q_{j}\right\rangle$ :

$$
\begin{aligned}
\mathcal{F}_{X Y}\left(\tilde{f}_{i j}\right)= & \sum_{L} \sum_{i j}\left\langle X^{*}\left(Q_{i_{1}} \cdots Q_{i_{L}}\right)\right\rangle \widetilde{f}_{i_{1} j_{1}} \cdots \widetilde{f}_{i_{L} j_{L}} \\
& \times\left\langle\left(Q_{j_{1}} \cdots Q_{j_{L}}\right)^{*} Y\right\rangle .
\end{aligned}
$$

If one uses the factorization theorem for the leading-order treatment of the $\beta$-relaxation window, $\Phi_{i j}(t)=f_{i j}^{c}$ $+h_{i j} G(t)$, one gets the corresponding result for the $X-Y$ correlator

$$
\Phi_{X Y}(t)=f_{X Y}^{c}+h_{X Y} G(t) .
$$

Here the critical nonergodicity parameter is given by the functional at the critical point, $f_{X Y}^{c}=\mathcal{F}_{X Y}\left(f_{i j}^{c}\right)$, and the critical amplitude is given by the functional's derivative: $h_{X Y}$ $=\Sigma_{i j}\left[\partial \mathcal{F}_{X Y}\left(f_{i j}^{c}\right) / \partial f_{i j}\right] h_{i j}$. Higher-order powers in $G(t)$ contribute to the corrections to the leading-order $\beta$-relaxation result.

According to Eq. (46) it is obvious within MCT that all correlators of variables $X$ and $Y$ that couple to densities, i.e., for which not all products of overlaps $\left\langle X^{*}\left(Q_{i_{1}} \cdots Q_{i_{L}}\right)\right\rangle\left\langle\left(Q_{j_{1}} \cdots Q_{j_{L}}\right) * Y\right\rangle$ vanish, exhibit the uni- versal features for structural relaxation. For example, if one analyzes the depolarized-light-scattering cross sections within the leading-order MCT formulas [5,19,20], one can use the known expressions for $G(t)$, provided one treats $h_{X Y}, t_{0}, \sigma$, and $\lambda$-and only these numbers-as fit parameters. It is irrelevant in that context whether the scattering is caused primarily by reorientational contributions or not. Similarly, it is legitimate to analyze dielectric spectra with the MCT scaling-law results [12,13], independent of whether the response arises from ion displacements, from reorientations of dipole moments, or from a combination of both contributions. The formulas hold for the dielectric function as well as for the dielectric modulus. Our paper provides a firstprinciples theory for the amplitudes $f_{i j}^{c}(q \mu), h_{i j}(q \mu)$ in Eq. (44b).

The transient dynamics for reorientational motion deals with hindered rotation, libration, and orientational diffusion. The crossover from transient to structural relaxation cannot be handled by Eqs. (45) and (46). To attack this problem one needs a formalism that accounts reasonably for the transient motion of the molecule as well as for its structural relaxation. To deal with the transient, we have used the continuedfraction representations of the correlators in Sec. II D and to account for the structural relaxation, the MCT feedback ideas have been formulated for the relaxation kernels in Sec. III A. To handle both mentioned aspects of the molecule's dynamics, the mode-coupling ideas have not been applied directly for the reorientational correlators as suggested by Eqs. (45). Rather the factorization approximation has been introduced for the memory kernels determining these functions.

One can use the recently developed procedures to evaluate from the mode-coupling functionals the leading corrections to Eqs. (43) and (44b), which are specified by two sets of amplitudes $K_{i j}(q \nu), \bar{K}_{i j}(q \nu)$ [32]. These corrections extend the range of validity of the mentioned leading-order results and determine their range of validity. It is obvious, for example, that the range of validity for the dielectric loss spectrum may be different from that for the modulus. Work is in progress to determine the indicated results for our model and to compare theses findings with numerical solutions for the correlators and spectra.

\section{Type- $A$ transitions}

Let us imagine that for fixed $\varphi \geqslant \varphi_{c}$ the dumbbell size shrinks as described by a decrease of $d_{1}, d_{2}$, and $\zeta$. For properly chosen small $d_{1}, d_{2}$, there will obviously appear a critical value of $\zeta$, say $\hat{\zeta}_{c}(\varphi)$, so that the molecule can no longer be localized for $\zeta<\hat{\zeta}_{c}(\varphi)$. There will be a percolation transition for $\zeta$ crossing $\hat{\zeta}_{c}(\varphi)$. The $\zeta$ - $\varphi$ parameter plane will split into three regions. For $\varphi<\varphi_{c}$ there is an ergodic liquid with a diffusing molecule. For $\varphi \geqslant \varphi_{c}$ and $\zeta>\bar{\zeta}_{c}(\varphi)$ there is a glass with a localized molecule, and for $\zeta<\hat{\zeta}_{c}(\varphi)$ there is a glass with a delocalized molecule. MCT brings out a type$A$ transition for $\zeta$ crossing a percolation threshold $\hat{\zeta}_{c}(\varphi)$ as demonstrated before for the Lorentz system $[55,56]$. The topology of the phase diagram is that discussed by Sjögren [65] for a schematic MCT model. At the percolation threshold the particle localization length diverges. The approximations underlying the MCT cannot exactly handle the perco- 
lation transition, whose universal dynamics is connected with a divergent length scale. Indeed, the asymptotic MCT results $[55,56]$ are those of a mean-field approach.

Type- $A$ transitions for rotational degrees of freedom have been studied by Michel for crystals containing nonspherical molecules as impurities [66]. His assumed frozen random array of impurity positions is the analog of our glass environment. His general equations of motion are similar to ours; but his problem is more complicated due to the impurityimpurity interactions. Generically, interacting systems cannot exhibit a type- $A$ transition. Such transitions are possible only if there is some symmetry in the problem, rendering whole blocks of mode-coupling vertices zero, or if the block structure is due to some peculiarity of the model [57]. The latter is the case for the percolation problem: the frozen surroundings govern the dynamics of the molecule but the single molecule cannot influence the ergodicity properties of the surroundings. In Ref. [66] it is anticipated that the impurity-impurity mode-coupling interactions are so small that the expected jump of the nonergodicity parameter at the transition can be ignored, and thereby a type- $B$ transition is approximated by a type- $A$ one.

If one considers a top-down symmetric molecule within our theory, a type- $A$ transition for odd $-j$ correlators is possible even though the even- $j$ correlators are arrested. The glass state for a localized molecule with arrested even- $j$ dynamics splits into two pieces, separated by a $\zeta_{c}(\varphi)$ transition line. For $\zeta<\zeta_{c}(\varphi)$, there is a glass with ergodic odd- $j$ dynamics of the molecule and for $\zeta>\zeta_{c}(\varphi)$ all rotational variables are arrested. Figure 5 exhibits the continuous decrease of the odd $-j$ nonergodicity parameters for $\zeta$ approaching $\zeta_{c}$ $=\zeta_{c}\left(\varphi_{c}\right)$ from above. With increasing $\varphi$ the trend to arrest increases, and therefore $\zeta_{c}(\varphi)$ for $\varphi>\varphi_{c}$ is smaller than $\zeta_{\dot{c}}$, as is demonstrated in Fig. 7. Let us emphasize that the described odd- $j$ transition is not connected with a divergent length scale. One finds that the nonergodicity parameters increase linearly with increasing separation parameter: $f(q j \mu)=\widetilde{h}(q j \mu)\left(\zeta-\zeta_{c}\right)+O\left(\left(\zeta-\zeta_{c}\right)^{2}\right)$ for $\zeta \geqslant \zeta_{c}$. This linear variation is demonstrated in Fig. 5 and, on a magnified abscissa, in Fig. 9. If the symmetry is slightly broken, the transition is eliminated in favor of a rapid crossover from very small nonergodicity parameters for $\zeta \ll \zeta_{c}$ to such of order unity for $\zeta \gg \zeta_{c}$ [57], as is also shown in Fig. 9. The crossover dynamics can be described by a formula such as Eq. (46), which specifies universality features.

\section{ACKNOWLEDGMENTS}

We are very indebted to Herman Z. Cummins for many stimulating discussions and for constructive criticism. We acknowledge helpful hints from Karl H. Michel. We thank R. Schilling for discussions, comments on our manuscript, and information on his studies of molecular liquids. Our work was supported by Verbundprojekt BMBF 03G04TUM.

\section{APPENDIX: THE MODE-COUPLING FUNCTIONAL}

In this appendix, the details of the mode-coupling functional $\mathcal{F}$ in Eq. (30c) shall be specified. From Eqs. (16a) and

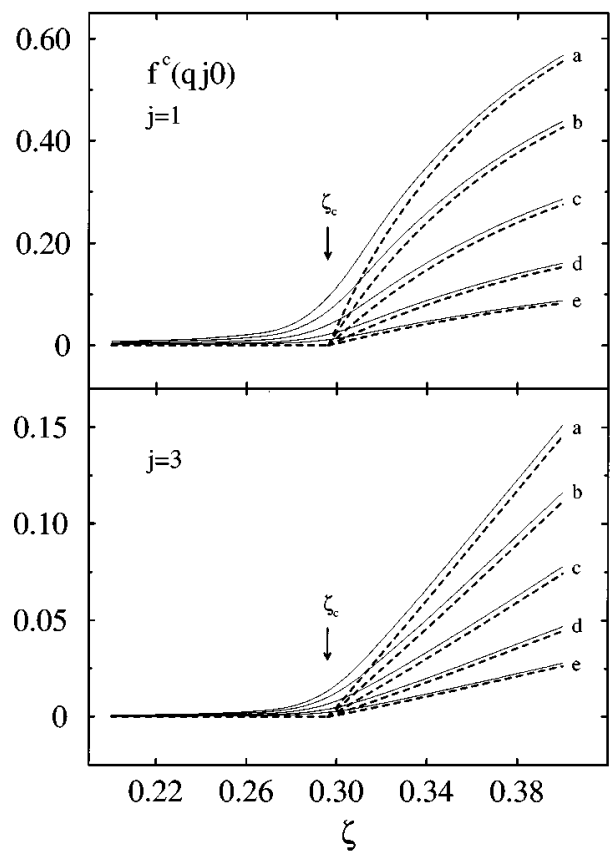

FIG. 9. Critical glass form factors $f^{c}(q j 0)$ for helicity zero as a function of $\zeta$ for $q=3.4$ (a), 7.0 (b), 10.6 (c), 14.2 (d), and 17.4 (e); $j=1$ and $j=3$. The dashed heavy lines refer to top-down symmetric dumbbells $d_{1}=d_{2}=d$; the arrows indicate the position $\zeta_{c}=0.296$ for a type- $A$ transition. The full light lines are the results for a molecule with $\left(d_{1}-d_{2}\right) / d=0.02, d_{2}=d$.

(29a) one gets as a starting formula

$$
\begin{aligned}
\mathcal{F}_{\alpha i \beta j q \mu}\left(\widetilde{f}_{l^{\prime} l}(k \nu) ; \widetilde{f}_{p}\right)= & \sum_{\vec{k} \vec{p} l^{\prime} l \nu}[N S(p)]^{-1} A_{l^{\prime}}(\vec{k} \vec{p} \nu ; \alpha i q \mu)^{*} \\
& \times \widetilde{f}_{l^{\prime} l}(k \nu) \widetilde{f}_{p} A_{l}(\vec{k} \vec{p} \nu ; \beta j q \mu), \quad(\mathrm{A} 1)
\end{aligned}
$$

with

$$
A_{l}(\vec{k} \vec{p} \nu ; \beta j q \mu)=\sum_{\kappa} D_{\kappa \nu}^{l}(\vec{k})\left(A_{l}^{\kappa}(\vec{k} \vec{p}) \mid F_{\beta j}(q \mu)\right) \sqrt{N S(p)}
$$

There are two contributions to the overlap of fluctuating forces $F$ and pair modes $A$. One is due to the last term in Eq. (26), which yields with abbreviation (13)

$$
\begin{aligned}
& \left\langle\varrho_{l}^{\kappa}(\vec{k})^{*} \varrho(\vec{p})^{*} \varrho_{j}^{\mu}\left(\vec{q}_{0}\right)\right\rangle \\
& \quad=\left\langle\varrho(\vec{p})^{*} \pi_{j l}^{\mu-\kappa}\left(\vec{q}_{0},-\vec{k}, O\right)\right\rangle(-1)^{l+\kappa} .
\end{aligned}
$$

The other one is due to the first term on the rhs of Eq. (26). It can be reformulated in a similar manner if one uses Kubo's identity $\left\langle A^{*} \mathcal{L} B\right\rangle=i T\left\langle\left\{B, A^{*}\right\}\right\rangle:\left\langle\varrho_{l}^{\kappa}(\vec{k})^{*} \varrho(\vec{p})^{*} \mathcal{L} \sigma_{\beta j \mu}\left(\vec{q}_{0}\right)\right\rangle$ $=-T\left\langle\varrho(\vec{p})^{*} \pi_{j l}^{\mu-\kappa}\left(\vec{q}_{0},-\vec{k}, \beta\right)\right\rangle(-1)^{l+\kappa}$. The remaining averages have been specified in Eqs. (14) and hence 


$$
\begin{aligned}
& A_{l}(\vec{k} \vec{p} \nu ; \beta j q \mu)=\delta_{\vec{p}+\vec{k}, \vec{q}_{0}}[4 \pi(2 j+1)(2 l+1)]^{1 / 2} \sum_{\kappa J M} \mathrm{i}^{j+l-J}(-1)^{l+\kappa+1} Y_{J}^{M}(\vec{p})^{*} n S(p) c_{J}(p)\left(\begin{array}{ccc}
j & l & J \\
\mu & -\kappa & M
\end{array}\right) \\
& \times\left(\begin{array}{ccc}
j & l & J \\
0 & 0 & 0
\end{array}\right) D_{\kappa \nu}^{l}(\vec{k})\left[C_{\beta}\left(j l J ; \vec{q}_{0} \cdot(-\vec{k})\right) v_{\beta}^{2} / \Omega_{\beta j}(q)^{2}+C_{\mathrm{O}}\right] .
\end{aligned}
$$

The $\vec{k}-\vec{p}$ sum in Eq. (A1) reduces to an integration over $\vec{k}$, since $\vec{p}=\vec{q}_{0}-\vec{k}$. This is done best in bipolar coordinates: $\left[(2 \pi)^{3} / N\right] \Sigma_{\vec{k} \vec{p}} \delta_{\vec{p}+\vec{k}, \vec{q}_{0}} \cdots=1 /(n q) \int d \varphi \int k d k \int p d p \cdots$. Here $\left(\varphi, \vartheta_{k}\right)$ denotes the solid angle of $\vec{k}$, so that $\cos \vartheta_{k}=\left(k^{2}-p^{2}\right.$ $\left.+q^{2}\right) /(2 k q)$. The solid angle of $\vec{p}$ is $\left(-\varphi, \vartheta_{p}\right)$ with $\cos \vartheta_{p}=\left(p^{2}-k^{2}+q^{2}\right) /(2 p q)$. The integration over $\varphi$ yields

$$
2 \int Y_{J^{\prime}}^{M^{\prime}}(\vec{p}) D_{\kappa^{\prime} \nu}^{l^{\prime}}(\vec{k})^{*} Y_{J}^{M}(\vec{p})^{*} D_{\kappa \nu}^{l}(\vec{k}) d \varphi=\delta_{M^{\prime}-M, \kappa^{-} \kappa^{\prime}} \sqrt{\left(2 J^{\prime}+1\right)(2 J+1)} d_{M^{\prime} 0}^{J^{\prime}}\left(\vartheta_{p}\right) d_{M 0}^{J}\left(\vartheta_{p}\right) d_{\kappa^{\prime} \nu}^{l^{\prime}}\left(\vartheta_{k}\right) d_{\kappa \nu}^{l}\left(\vartheta_{k}\right) .
$$

The Kronecker symbol and the 3-j symbols together imply $M^{\prime}=M$ and $\kappa^{\prime}=\kappa$. Finally, the integrals are replaced by Riemann sums of $N_{q}$ wave-vector moduli, equally spaced with distance $h: k / h, p / h=1 / 2,3 / 2, \ldots,\left(N_{q}-1 / 2\right)$. This yields the expression for the coefficients in Eq. (30c), where the sum over $p$ is restricted to $|q-k| \leqslant p \leqslant|q+k|$ :

$$
\begin{aligned}
& V_{q \mu k p l^{\prime} l \nu}^{\alpha i \beta j}=\frac{1}{(4 \pi)^{2}} \frac{h^{2} k p}{q} \sqrt{(2 i+1)(2 j+1)\left(2 l^{\prime}+1\right)(2 l+1)} \\
& \times \sum_{J^{\prime} J} n S(p) c_{J^{\prime}}(p) c_{J}(p) \sqrt{\left(2 J^{\prime}+1\right)(2 J+1)}\left(\begin{array}{ccc}
i & l^{\prime} & J^{\prime} \\
0 & 0 & 0
\end{array}\right)\left(\begin{array}{ccc}
j & l & J \\
0 & 0 & 0
\end{array}\right)(-1)^{\left(i-l^{\prime}-J^{\prime}\right) / 2} \\
& \times(-1)^{(j-l-J) / 2} \Delta_{\alpha}\left(i l^{\prime} J^{\prime}, q k p\right) \Delta_{\beta}(j l J, q k p) \sum_{\kappa} d_{\kappa-\mu 0}^{J^{\prime}}\left(\vartheta_{p}\right) d_{\kappa-\mu 0}^{J}\left(\vartheta_{p}\right) d_{\kappa \nu}^{l^{\prime}}\left(\vartheta_{k}\right) d_{\kappa \nu}^{l}\left(\vartheta_{k}\right)\left(\begin{array}{ccc}
i & l^{\prime} & J^{\prime} \\
\mu & -\kappa & \kappa-\mu
\end{array}\right) \\
& \times\left(\begin{array}{ccc}
j & l & J \\
\mu & -\kappa & \kappa-\mu
\end{array}\right),
\end{aligned}
$$

with the abbreviation

$$
\Delta_{\beta}(j l J, q k p)=\left\{\begin{array}{cc}
\left(p^{2}+q^{2}-k^{2}\right) / q^{2} & \text { if } \beta=T \\
{[J(J+1)+j(j+1)-l(l+1)] /[j(j+1)]} & \text { if } \beta=R
\end{array}\right.
$$

The angular momentum indices $j$ are taken into account up to and including a cutoff number $j_{\max }$. The results as shown in Figs. 2, 6, 7, 8 were calculated with $N_{q}=100, j_{\max }=6$, and the wave-vector spacing is chosen as $h=0.4$. Restricting the work to $j_{\max }=4$ changes the results on a $5 \%$ level for $j=0,1,2$ and on a $10 \%$ level for $j=3,4$. Using $j_{\max }=2$ one obtains results with a $20 \%$ error. Figures 3, 4, 5, and 9 are calculated with $j_{\max }=4$. Refining the discretization from $N_{q}=100$ to $N_{q}=200$ with the same large-wave-vector cutoff does not change the results on a $0.5 \%$ level, except for small $q$. Figures 3 and 4 are evaluated with $N_{q}=200$. Using $N_{q}=80$ instead of $N_{q}=100$ with identical spacing $h$ influences the results by less than $0.5 \%$, except for wave-vector moduli near the large-wave-vector cutoff, where results change on a 5\% level. Figures 5 and 9 are evaluated with $N_{q}=80$.

[1] J. Wong and C. A. Angell, Glass: Structure by Spectroscopy (Marcel Dekker, Inc., New York, 1976).

[2] J.-P. Boon and S. Yip, Molecular Hydrodynamics (McGrawHill, New York, 1980).

[3] J.-P. Hansen and I. R. McDonald, Theory of Simple Liquids, 2nd ed. (Academic Press, London, 1986).

[4] U. Balucani and M. Zoppi, Dynamics of the Liquid State (Clarendon Press, Oxford, 1994).

[5] H. Z. Cummins, G. Li, W. Du, Y. H. Hwang, and G. Q. Shen, Prog. Theor. Phys. Suppl. 126, 21 (1997).

[6] J. Wuttke, J. Hernandez, G. Li, G. Coddens, H. Z. Cummins, F. Fujara, W. Petry, and H. Sillescu, Phys. Rev. Lett. 72, 3052 (1994).
[7] J. Wuttke, W. Petry, G. Coddens, and F. Fujara, Phys. Rev. E 52, 4026 (1995).

[8] Y. Yang and K. A. Nelson, Phys. Rev. Lett. 74, 4883 (1995).

[9] Y. Yang and K. A. Nelson, J. Chem. Phys. 103, 7732 (1995).

[10] Y. Yang and K. A. Nelson, Mater. Res. Soc. Symp. Proc. 407, 147 (1996).

[11] J. Ma, D. V. Bout, and M. Berg, Phys. Rev. E 54, 2786 (1996).

[12] P. Lunkenheimer, A. Pimenov, M. Dressel, Y. G. Goncharov, R. Böhmer, and A. Loidl, Phys. Rev. Lett. 77, 318 (1996).

[13] P. Lunkenheimer, A. Pimenov, M. Dressel, B. Schreier, U. Schneider, and A. Loidl, Prog. Theor. Phys. Suppl. 126, 123 (1997).

[14] W. Kob and H. C. Andersen, Phys. Rev. E 51, 4626 (1995). 
[15] W. Kob and H. C. Andersen, Phys. Rev. E 52, 4134 (1995).

[16] F. Sciortino, P. Gallo, P. Tartaglia, and S.-H. Chen, Phys. Rev. E 54, 6331 (1996).

[17] G. Wahnström and J. Lewis, Prog. Theor. Phys. Suppl. 126, 261 (1997)

[18] W. Knaak, F. Mezei, and B. Farago, Europhys. Lett. 7, 529 (1988).

[19] N. J. Tao, G. Li, and H. Z. Cummins, Phys. Rev. Lett. 66, 1334 (1991).

[20] G. Li, W. M. Du, X. K. Chen, H. Z. Cummins, and N. J. Tao, Phys. Rev. A 45, 3867 (1992).

[21] E. Bartsch, F. Fujara, J. F. Legrand, W. Petry, H. Sillescu, and J. Wuttke, Phys. Rev. E 52, 738 (1995).

[22] M. Goldstein, J. Chem. Phys. 51, 3728 (1969).

[23] W. van Megen and S. M. Underwood, Phys. Rev. Lett. 70, 2766 (1993).

[24] W. van Megen and S. M. Underwood, Phys. Rev. E 49, 4206 (1994).

[25] E. Bartsch, J. Non-Cryst. Solids 192-193, 384 (1995).

[26] T. G. Mason and D. A. Weitz, Phys. Rev. Lett. 75, 2770 (1995).

[27] L. Sjögren, Phys. Rev. A 22, 2866 (1980).

[28] U. Bengtzelius, W. Götze, and A. Sjölander, J. Phys. C 17, 5915 (1984).

[29] W. Götze and L. Sjögren, Transp. Theory Stat. Phys. 24, 801 (1995).

[30] C. Z.-W. Liu and I. Oppenheim, Physica A 235, 369 (1997).

[31] S. F. Edwards and P. W. Anderson, J. Phys. F 5, 965 (1975).

[32] T. Franosch, M. Fuchs, W. Götze, M. R. Mayr, and A. P. Singh, Phys. Rev. E 55, 7153 (1997).

[33] W. Götze and L. Sjögren, Rep. Prog. Phys. 55, 241 (1992).

[34] R. Schilling, in Disorder Effects on Relaxational Processes, edited by R. Richert and A. Blumen (Springer-Verlag, Berlin, 1994), p. 193.

[35] S. P. Das and G. F. Mazenko, Phys. Rev. A 34, 2265 (1986).

[36] W. Götze and L. Sjögren, Z. Phys. B 65, 415 (1987).

[37] M. Fuchs, W. Götze, S. Hildebrand, and A. Latz, J. Phys.: Condens. Matter 4, 7709 (1992).

[38] W. van Megen and S. Underwood, Phys. Rev. Lett. 72, 1773 (1994).

[39] W. van Megen, Transp. Theory Stat. Phys. 24, 1017 (1995).

[40] M. Nauroth and W. Kob, Phys. Rev. E 55, 657 (1997).
[41] H. Z. Cummins, W. M. Du, M. Fuchs, W. Götze, S. Hildebrand, A. Latz, G. Li, and N. J. Tao, Phys. Rev. E 47, 4223 (1993).

[42] M. Evans, G. J. Evans, W. T. Coffey, and P. Grigolini, Molecular Dynamics and Theory of Broad Band Spectroscopy (John Wiley \& Sons, New York, 1982).

[43] W. A. Steele, J. Chem. Phys. 39, 3197 (1963).

[44] L. Blum and A. J. Torruella, J. Chem. Phys. 56, 303 (1972).

[45] R. Schilling and T. Scheidsteger, Phys. Rev. E 56, 2932 (1997).

[46] D. Forster, Hydrodynamic Fluctuations, Broken Symmetry, and Correlation Functions (W. A. Benjamin, Reading, MA, 1975).

[47] A. Messiah, Quantum Mechanics (North-Holland, Amsterdam, 1962).

[48] W. Götze, Solid State Commun. 27, 1393 (1978).

[49] W. Götze, J. Phys. C 12, 1279 (1979).

[50] W. Götze, Philos. Mag. B 43, 219 (1981).

[51] K. Kawasaki, Phys. Rev. 150, 291 (1966).

[52] K. Kawasaki, Ann. Phys. (N.Y.) 61, 1 (1970).

[53] A. J. Masters and T. Keyes, J. Stat. Phys. 39, 215 (1985).

[54] R. D. Mountain, J. Res. Natl. Bur. Stand. 70A, 207 (1966).

[55] W. Götze, E. Leutheusser, and S. Yip, Phys. Rev. A 23, 2634 (1981).

[56] W. Götze, E. Leutheusser, and S. Yip, Phys. Rev. A 24, 1008 (1981).

[57] T. Franosch and W. Götze, J. Phys. Condens. Matter 6, 4807 (1994).

[58] E. Leutheusser, Phys. Rev. A 29, 2765 (1984).

[59] J.-L. Barrat and A. Latz, J. Phys. Condens. Matter 2, 4289 (1990).

[60] M. Fuchs and A. Latz, Physica A 201, 1 (1993).

[61] T. Franosch and A. P. Singh, J. Chem. Phys. 107, 5524 (1997).

[62] M. Fuchs, I. Hofacker, and A. Latz, Phys. Rev. A 45, 898 (1992).

[63] G. Wahnström (private communication).

[64] W. Götze, in Liquids, Freezing and Glass Transition, edited by J. P. Hansen, D. Levesque, and J. Zinn-Justin (North-Holland, Amsterdam, 1991), p. 287.

[65] L. Sjögren, Phys. Rev. A 33, 1254 (1986).

[66] K. H. Michel, Z. Phys. B 68, 259 (1987). 